EUROPEAN ORGANIZATION FOR NUCLEAR RESEARCH (CERN)

CERN-EP/2001-022

February 28, 2001

\title{
Measurement of Triple Gauge-Boson Couplings at LEP energies up to $189 \mathrm{GeV}$
}

\author{
The ALEPH Collaboration*)
}

\begin{abstract}
The triple gauge-boson couplings involving the $\mathrm{W}$ are determined using data samples collected with the ALEPH detector at mean centre-of-mass energies of $183 \mathrm{GeV}$ and $189 \mathrm{GeV}$, corresponding to integrated luminosities of $57 \mathrm{pb}^{-1}$ and $174 \mathrm{pb}^{-1}$, respectively. The couplings, $\mathrm{g}_{1}^{\mathrm{Z}}, \kappa_{\gamma}$ and $\lambda_{\gamma}$, are measured using $\mathrm{W}$-pair events, single$\mathrm{W}$ production and single- $\gamma$ production. Each coupling is measured individually with the other two couplings fixed at their Standard Model value. Including ALEPH results from lower energies, the $95 \%$ confidence level intervals for the deviation to the Standard Model are

$$
\begin{aligned}
& -0.087<\Delta \mathrm{g}_{1}^{\mathrm{Z}}<0.141 \\
& -0.200<\Delta \kappa_{\gamma}<0.258 \\
& -0.062<\lambda_{\gamma}<0.147 .
\end{aligned}
$$

Fits are also presented where two or all three couplings are allowed to vary. In addition, W-pair events are used to set limits on the C- or P-violating couplings $\mathrm{g}_{4}^{\mathrm{V}}$, $\mathrm{g}_{5}^{\mathrm{V}}, \tilde{\kappa}_{\mathrm{V}}$, and $\tilde{\lambda}_{\mathrm{V}}$, where $\mathrm{V}$ denotes either $\gamma$ or Z. No deviations from the Standard Model expectations are observed.
\end{abstract}

(Submitted to European Physical Journal C)

*) See next pages for the list of authors. 


\section{The ALEPH Collaboration}

A. Heister, S. Schael

Physikalisches Institut das RWTH-Aachen, D-52056 Aachen, Germany

R. Barate, I. De Bonis, D. Decamp, P. Ghez, C. Goy, S. Jezequel, J.-P. Lees, F. Martin, E. Merle, M.-N. Minard, B. Pietrzyk, B. Trocmé Laboratoire de Physique des Particules (LAPP), IN² P3_CNRS, F-74019 Annecy-le-Vieux Cedex, France

S. Bravo, M.P. Casado, M. Chmeissani, J.M. Crespo, E. Fernandez, M. Fernandez-Bosman, Ll. Garrido, ${ }^{15}$

E. Graugés, J. Lopez, M. Martinez, G. Merino, R. Miquel, Ll.M. Mir, A. Pacheco, D. Paneque, H. Ruiz Institut de Física d'Altes Energies, Universitat Autònoma de Barcelona, E-08193 Bellaterra (Barcelona), Spain ${ }^{7}$

A. Colaleo, D. Creanza, N. De Filippis, M. de Palma, G. Iaselli, G. Maggi, M. Maggi, ${ }^{1}$ S. Nuzzo, A. Ranieri, G. Raso ${ }^{24}$ F. Ruggieri, G. Selvaggi, L. Silvestris, P. Tempesta, A. Tricomi ${ }^{3}$ G. Zito

Dipartimento di Fisica, INFN Sezione di Bari, I-70126 Bari, Italy

X. Huang, J. Lin, Q. Ouyang, T. Wang, Y. Xie, R. Xu, S. Xue, J. Zhang, L. Zhang, W. Zhao Institute of High Energy Physics, Academia Sinica, Beijing, The People's Republic of China ${ }^{8}$

D. Abbaneo, P. Azzurri, T. Barklow, ${ }^{30}$ G. Boix ${ }^{6}$ O. Buchmüller, M. Cattaneo, F. Cerutti, B. Clerbaux, G. Dissertori, H. Drevermann, R.W. Forty, M. Frank, F. Gianotti, T.C. Greening, J.B. Hansen, J. Harvey, D.E. Hutchcroft, P. Janot, B. Jost, M. Kado, V. Lemaitre, ${ }^{23}$ P. Maley, P. Mato, A. Moutoussi, F. Ranjard, L. Rolandi, D. Schlatter, P. Spagnolo, W. Tejessy, F. Teubert, E. Tournefier, ${ }^{26}$ A. Valassi, J.J. Ward, A.E. Wright

European Laboratory for Particle Physics (CERN), CH-1211 Geneva 23, Switzerland

Z. Ajaltouni, F. Badaud, S. Dessagne, A. Falvard, ${ }^{20}$ D. Fayolle, P. Gay, P. Henrard, J. Jousset, B. Michel,

S. Monteil, J-C. Montret, D. Pallin, J.M. Pascolo, P. Perret, F. Podlyski

Laboratoire de Physique Corpusculaire, Université Blaise Pascal, IN $\mathrm{N}^{2} \mathrm{P}^{3}$ CNRS, Clermont-Ferrand, F-63177 Aubière, France

J.D. Hansen, J.R. Hansen, P.H. Hansen, B.S. Nilsson, A. Wäänänen

Niels Bohr Institute, 2100 Copenhagen, DK-Denmark ${ }^{9}$

G. Daskalakis, A. Kyriakis, C. Markou, E. Simopoulou, A. Vayaki

Nuclear Research Center Demokritos (NRCD), GR-15310 Attiki, Greece

A. Blondel, ${ }^{12}$ J.-C. Brient, $\quad$ F. Machefert, A. Rougé, M. Swynghedauw, R. Tanaka H. Videau

Laboratoire de Physique Nucléaire et des Hautes Energies, Ecole Polytechnique, $I N^{2} P^{3}-C N R S$, F-91128 Palaiseau Cedex, France

E. Focardi, G. Parrini, K. Zachariadou

Dipartimento di Fisica, Università di Firenze, INFN Sezione di Firenze, I-50125 Firenze, Italy

A. Antonelli, M. Antonelli, G. Bencivenni, G. Bologna, ${ }^{4}$ F. Bossi, P. Campana, G. Capon, V. Chiarella, P. Laurelli, G. Mannocchi, ${ }^{5}$ F. Murtas, G.P. Murtas, L. Passalacqua, M. Pepe-Altarelli ${ }^{25}$

Laboratori Nazionali dell'INFN (LNF-INFN), I-00044 Frascati, Italy

M. Chalmers, A.W. Halley, J. Kennedy, J.G. Lynch, P. Negus, V. O'Shea, B. Raeven, D. Smith, A.S. Thompson

Department of Physics and Astronomy, University of Glasgow, Glasgow G12 8QQ,United Kingdom ${ }^{10}$

S. Wasserbaech

Department of Physics, Haverford College, Haverford, PA 19041-1392, U.S.A. 
R. Cavanaugh, S. Dhamotharan, C. Geweniger, P. Hanke, V. Hepp, E.E. Kluge, G. Leibenguth, A. Putzer, K. Tittel, S. Werner, ${ }^{19}$ M. Wunsch ${ }^{19}$

Kirchhoff-Institut für Physik, Universität Heidelberg, D-69120 Heidelberg, Germany ${ }^{16}$

R. Beuselinck, D.M. Binnie, W. Cameron, G. Davies, P.J. Dornan, M. Girone, ${ }^{1}$ N. Marinelli, J. Nowell,

H. Przysiezniak, ${ }^{2}$ S. Rutherford, J.K. Sedgbeer, J.C. Thompson, ${ }^{14}$ R. White

Department of Physics, Imperial College, London SW7 2BZ, United Kingdom ${ }^{10}$

V.M. Ghete, P. Girtler, E. Kneringer, D. Kuhn, G. Rudolph

Institut für Experimentalphysik, Universität Innsbruck, A-6020 Innsbruck, Austria ${ }^{18}$

E. Bouhova-Thacker, C.K. Bowdery, D.P. Clarke, G. Ellis, A.J. Finch, F. Foster, G. Hughes, R.W.L. Jones, ${ }^{1}$

M.R. Pearson, N.A. Robertson, M. Smizanska

Department of Physics, University of Lancaster, Lancaster LA1 4YB, United Kingdom ${ }^{10}$

I. Giehl, F. Hölldorfer, K. Jakobs, K. Kleinknecht, M. Kröcker, A.-S. Müller, H.-A. Nürnberger, G. Quast,

B. Renk, E. Rohne, H.-G. Sander, S. Schmeling, H. Wachsmuth, C. Zeitnitz, T. Ziegler

Institut für Physik, Universität Mainz, D-55099 Mainz, Germany ${ }^{16}$

A. Bonissent, J. Carr, P. Coyle, C. Curtil, A. Ealet, D. Fouchez, O. Leroy, T. Kachelhoffer, P. Payre,

D. Rousseau, A. Tilquin

Centre de Physique des Particules de Marseille, Univ Méditerranée, $I N^{2} P^{3}$-CNRS, F-13288 Marseille, France

M. Aleppo, S. Gilardoni, F. Ragusa

Dipartimento di Fisica, Università di Milano e INFN Sezione di Milano, I-20133 Milano, Italy.

A. David, H. Dietl, G. Ganis, ${ }^{27}$ K. Hüttmann, G. Lütjens, C. Mannert, W. Männer, H.-G. Moser, R. Settles, ${ }^{1}$

H. Stenzel, G. Wolf

Max-Planck-Institut für Physik, Werner-Heisenberg-Institut, D-80805 München, Germany

J. Boucrot, ${ }^{1}$ O. Callot, M. Davier, L. Duflot, J.-F. Grivaz, Ph. Heusse, A. Jacholkowska, ${ }^{20}$ L. Serin, J.-J. Veillet, I. Videau, J.-B. de Vivie de Régie, ${ }^{28}$ C. Yuan

Laboratoire de l'Accélérateur Linéaire, Université de Paris-Sud, $I N^{2} P^{3}$-CNRS, F-91898 Orsay Cedex, France

G. Bagliesi, T. Boccali, G. Calderini, V. Ciulli, L. Foà, A. Giammanco, A. Giassi, F. Ligabue, A. Messineo,

F. Palla, G. Sanguinetti, A. Sciabà, G. Sguazzoni, R. Tenchini, ${ }^{1}$ A. Venturi, P.G. Verdini

Dipartimento di Fisica dell'Università, INFN Sezione di Pisa, e Scuola Normale Superiore, I-56010

Pisa, Italy

O. Awunor, G.A. Blair, J. Coles, G. Cowan, A. Garcia-Bellido, M.G. Green, L.T. Jones, T. Medcalf,

A. Misiejuk, J.A. Strong, P. Teixeira-Dias

Department of Physics, Royal Holloway \& Bedford New College, University of London, Egham, Surrey

TW20 OEX, United Kingdom ${ }^{10}$

R.W. Clifft, T.R. Edgecock, P.R. Norton, I.R. Tomalin

Particle Physics Dept., Rutherford Appleton Laboratory, Chilton, Didcot, Oxon OX11 OQX, United Kingdom ${ }^{10}$

B. Bloch-Devaux, ${ }^{1}$ D. Boumediene, P. Colas, B. Fabbro, E. Lançon, M.-C. Lemaire, E. Locci, P. Perez, J. Rander, J.-F. Renardy, A. Rosowsky, P. Seager, ${ }^{13}$ A. Trabelsi, ${ }^{21}$ B. Tuchming, B. Vallage

CEA, DAPNIA/Service de Physique des Particules, CE-Saclay, F-91191 Gif-sur-Yvette Cedex, France $^{17}$

N. Konstantinidis, A.M. Litke, C. Loomis, G. Taylor

Institute for Particle Physics, University of California at Santa Cruz, Santa Cruz, CA 95064, USA ${ }^{22}$

C.N. Booth, S. Cartwright, F. Combley, P.N. Hodgson, M. Lehto, L.F. Thompson

Department of Physics, University of Sheffield, Sheffield S3 7RH, United Kingdom ${ }^{10}$

K. Affholderbach, A. Böhrer, S. Brandt, C. Grupen, J. Hess, A. Ngac, G. Prange, U. Sieler 
Fachbereich Physik, Universität Siegen, D-57068 Siegen, Germany ${ }^{16}$

C. Borean, G. Giannini

Dipartimento di Fisica, Università di Trieste e INFN Sezione di Trieste, I-34127 Trieste, Italy

H. He, J. Putz, J. Rothberg

Experimental Elementary Particle Physics, University of Washington, Seattle, WA 98195 U.S.A.

S.R. Armstrong, K. Cranmer, P. Elmer, D.P.S. Ferguson, Y. Gao, ${ }^{29}$ S. González, O.J. Hayes, H. Hu, S. Jin, J. Kile, P.A. McNamara III, J. Nielsen, W. Orejudos, Y.B. Pan, Y. Saadi, I.J. Scott, J.H. von Wimmersperg-Toeller, J. Walsh, W. Wiedenmann, J. Wu, Sau Lan Wu, X. Wu, G. Zobernig

Department of Physics, University of Wisconsin, Madison, WI 53706, USA ${ }^{11}$

\footnotetext{
${ }^{1}$ Also at CERN, 1211 Geneva 23, Switzerland.

${ }^{2}$ Now at LAPP, 74019 Annecy-le-Vieux, France

${ }^{3}$ Also at Dipartimento di Fisica di Catania and INFN Sezione di Catania, 95129 Catania, Italy.

${ }^{4}$ Deceased.

${ }^{5}$ Also Istituto di Cosmo-Geofisica del C.N.R., Torino, Italy.

${ }^{6}$ Supported by the Commission of the European Communities, contract ERBFMBICT982894.

${ }^{7}$ Supported by CICYT, Spain.

${ }^{8}$ Supported by the National Science Foundation of China.

${ }^{9}$ Supported by the Danish Natural Science Research Council.

${ }^{10}$ Supported by the UK Particle Physics and Astronomy Research Council.

${ }^{11}$ Supported by the US Department of Energy, grant DE-FG0295-ER40896.

${ }^{12}$ Now at Departement de Physique Corpusculaire, Université de Genève, 1211 Genève 4, Switzerland.

${ }^{13}$ Supported by the Commission of the European Communities, contract ERBFMBICT982874.

${ }^{14}$ Also at Rutherford Appleton Laboratory, Chilton, Didcot, UK.

${ }^{15}$ Permanent address: Universitat de Barcelona, 08208 Barcelona, Spain.

${ }^{16}$ Supported by the Bundesministerium für Bildung, Wissenschaft, Forschung und Technologie, Germany.

${ }^{17}$ Supported by the Direction des Sciences de la Matière, C.E.A.

${ }^{18}$ Supported by the Austrian Ministry for Science and Transport.

${ }^{19}$ Now at SAP AG, 69185 Walldorf, Germany

${ }^{20}$ Now at Groupe d' Astroparticules de Montpellier, Université de Montpellier II, 34095 Montpellier, France.

${ }^{21}$ Now at Département de Physique, Faculté des Sciences de Tunis, 1060 Le Belvédère, Tunisia.

${ }^{22}$ Supported by the US Department of Energy, grant DE-FG03-92ER40689.

${ }^{23}$ Now at Institut de Physique Nucléaire, Département de Physique, Université Catholique de Louvain, 1348 Louvain-la-Neuve, Belgium.

${ }^{24}$ Also at Dipartimento di Fisica e Tecnologie Relative, Università di Palermo, Palermo, Italy.

${ }^{25}$ Now at CERN, 1211 Geneva 23, Switzerland.

${ }^{26}$ Now at ISN, Institut des Sciences Nucléaires, 53 Av. des Martyrs, 38026 Grenoble, France.

${ }^{27}$ Now at INFN Sezione di Roma II, Dipartimento di Fisica, Università di Roma Tor Vergata, 00133 Roma,

${ }^{28}$ Now at Centre de Physique des Particules de Marseille, Univ Méditerranée, F-13288 Marseille, France.

${ }^{29}$ Also at Department of Physics, Tsinghua University, Beijing, The People's Republic of China.

${ }^{30}$ Also at SLAC, Stanford, CA 94309, U.S.A.
} Italy. 


\section{Introduction}

The existence of the triple gauge-boson couplings (TGC) is a direct consequence of the $\mathrm{SU}(2)_{\mathrm{L}} \times \mathrm{U}(1)_{\mathrm{Y}}$ gauge theory. The measurement of the TGCs represents a fundamental test of the non-Abelian nature of the Standard Model. The triple WW $\gamma$ and WWZ couplings have been studied at LEP in $e^{+} e^{-}$collisions at energies above the $\mathrm{W}$-pair production threshold, using direct $\mathrm{W}$-pair production $\left(e^{+} e^{-} \rightarrow \mathrm{W}^{+} \mathrm{W}^{-}\right)$[1,2], single-W production $\left(e^{+} e^{-} \rightarrow \mathrm{We \nu}\right)$ and single- $\gamma$ production $\left(e^{+} e^{-} \rightarrow \nu \bar{\nu} \gamma(\gamma)\right)$ [3 [5]. Measurements of the TGCs have also been made at the Tevatron from studies of di-boson production [6]. This paper presents new results for the TGCs from analyses of W-pair, single-W, and single- $\gamma$ final states using data recorded in 1997 and 1998 with the ALEPH detector. In 1997 and 1998 ALEPH recorded total integrated luminosities of $56.81 \mathrm{pb}^{-1}$ and $174.20 \mathrm{pb}^{-1}$, at mean centre-of-mass energies of $182.66 \mathrm{GeV}$ and $188.63 \mathrm{GeV}$, denoted as 183 and $189 \mathrm{GeV}$.

The most general Lorentz invariant parametrisation of the $\mathrm{WW} \gamma$ and $\mathrm{WWZ}$ vertices can be described by 14 independent complex couplings $[7] 9], 7$ for each vertex: $g_{1}^{\mathrm{V}}, \mathrm{g}_{4}^{\mathrm{V}}$, $\mathrm{g}_{5}^{\mathrm{V}}, \kappa_{\mathrm{V}}, \lambda_{\mathrm{V}}, \tilde{\kappa}_{\mathrm{V}}$ and $\tilde{\lambda}_{\mathrm{V}}$, where $\mathrm{V}$ denotes either $\gamma$ or Z. Assuming electromagnetic gauge invariance, $\mathrm{C}$ - and P-conservation, the set of 14 couplings can be reduced to 5 parameters: $\mathrm{g}_{1}^{\mathrm{Z}}, \kappa_{\gamma}, \kappa_{\mathrm{Z}}, \lambda_{\gamma}$ and $\lambda_{\mathrm{Z}}$, with Standard Model values $\mathrm{g}_{1}^{\mathrm{Z}}=\kappa_{\mathrm{Z}}=\kappa_{\gamma}=1$ and $\lambda_{\mathrm{Z}}=\lambda_{\gamma}=0$. Precision measurements at the Z resonance at LEP and SLC also provide bounds on the couplings [10,11]. However, local $\mathrm{SU}(2)_{\mathrm{L}} \times \mathrm{U}(1)_{\mathrm{Y}}$ gauge invariance reduces the relevance of these bounds [10] and introduces the constraints:

$$
\begin{gathered}
\Delta \kappa_{\mathrm{Z}}=-\Delta \kappa_{\gamma} \tan ^{2} \theta_{\mathrm{w}}+\Delta \mathrm{g}_{1}^{\mathrm{Z}}, \\
\lambda_{\mathrm{Z}}=\lambda_{\gamma},
\end{gathered}
$$

where $\Delta$ denotes the deviation of the respective quantity from its non-zero Standard Model value, and $\theta_{\mathrm{W}}$ is the weak mixing angle. Hence, only three parameters remain: $\Delta \mathrm{g}_{1}^{\mathrm{Z}}, \Delta \kappa_{\gamma}$, and $\lambda_{\gamma}$ [9].

Using data from $e^{+} e^{-} \rightarrow \mathrm{W}^{+} \mathrm{W}^{-}$final states all three couplings $\Delta \mathrm{g}_{1}^{\mathrm{Z}}, \Delta \kappa_{\gamma}$ and $\lambda_{\gamma}$ can be tested, whereas the single- $W$ and single- $\gamma$ final states allow measurements of only the WW $\gamma$-couplings, $\Delta \kappa_{\gamma}$ and $\lambda_{\gamma}$. Although the contribution from W-pair production dominates the combined limits, the single- $\mathrm{W}$ and single- $\gamma$ events provide complementary information, which enhances the sensitivity especially for $\Delta \kappa_{\gamma}$.

In this analysis the three couplings $\Delta \mathrm{g}_{1}^{\mathrm{Z}}, \Delta \kappa_{\gamma}$ and $\lambda_{\gamma}$ are measured individually with the two other couplings fixed at zero, their Standard Model value. Fits are also presented, where two or all three couplings are allowed to vary.

The C- or P-violating sector of the TGCs is weakly bound. Indirect limits on $\tilde{\kappa}_{\gamma}$, $\tilde{\lambda}_{\gamma}, \tilde{\kappa}_{\mathrm{Z}}$ and $\mathrm{g}_{4}^{\mathrm{Z}}$ exist, while there are no direct or indirect limits on the parameters $\tilde{\lambda}_{\mathrm{Z}}$, $\mathrm{g}_{4}^{\gamma}, \mathrm{g}_{5}^{\gamma}$ and $\mathrm{g}_{5}^{\mathrm{Z}}$ [11]. Only the parameter $\tilde{\lambda}_{\gamma}$ is tightly constrained by precision low-energy measurements [12]. This paper includes, for the first time, single-parameter fits to the unconstrained real and imaginary parts of the 8 couplings $g_{4}^{\mathrm{V}}, \mathrm{g}_{5}^{\mathrm{V}}, \tilde{\kappa}_{\mathrm{V}}$, and $\tilde{\lambda}_{\mathrm{V}}$, all zero in

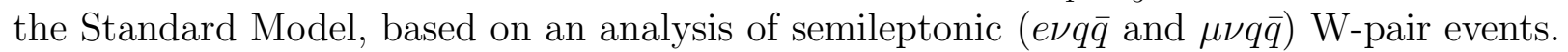

The paper is organised as follows. In Section 2, a brief description of the ALEPH detector is given. The Monte Carlo event generators used in the analyses are presented in Section 3 . The analysis of the single- $\gamma$ final states is described in Section 4 and Section 5 is devoted to the single-W analysis. The description of the two analyses is rather concise, as they are presented in earlier publications [3, 1 . In Section 6 the measurement of TGCs from W-pair 
events is discussed in detail. Finally, all measurements are combined with ALEPH results from $\mathrm{W}^{+} \mathrm{W}^{-}$production at $172 \mathrm{GeV}$ [1], single-W production at $183 \mathrm{GeV}$ [3] and single- $\gamma$ production at $183 \mathrm{GeV}$ [4]. The resulting single- and multi-parameter fits are discussed in Section 7, followed by a summary and conclusions in Section 8 .

\section{The ALEPH Detector}

A detailed description of the ALEPH detector can be found in Ref. [13, 14]. The central part of the ALEPH detector is dedicated to the reconstruction of the trajectories of charged particles. Following a charged particle from the interaction point outwards, the trajectory is measured by a two-layer silicon strip vertex detector (VDET), a cylindrical drift chamber and a large time projection chamber (TPC). The three tracking detectors are immersed in a $1.5 \mathrm{~T}$ axial field provided by a superconducting solenoidal coil. Altogether they measure charged particle momenta with a resolution of $\delta p_{\mathrm{T}} / p_{\mathrm{T}}=6 \times 10^{-4} p_{\mathrm{T}} \oplus 0.005\left(p_{\mathrm{T}}\right.$ in $\left.\mathrm{GeV} / \mathrm{c}\right)$. In the following, charged particle tracks reconstructed with at least one hit in the VDET, at least four hits in the TPC, and originating from within a cylinder of length $20 \mathrm{~cm}$ and 2 $\mathrm{cm}$ radius centred on the nominal interaction point and parallel with the beam, are referred to as good tracks.

Photons and electrons are identified in the electromagnetic calorimeter (ECAL), situated between the TPC and the coil. It is a lead-proportional-wire sampling calorimeter segmented in $0.9^{\circ} \times 0.9^{\circ}$ towers read out in three sections in depth. It has a total thickness of 22 radiation lengths and yields a relative energy resolution of $0.18 / \sqrt{E}+0.009$, with $E$ in $\mathrm{GeV}$, for isolated photons. At low polar angles, the ECAL is supplemented by two calorimeters, LCAL and SiCAL, principally used to measure the integrated luminosity collected by the experiment. Electrons are identified by their transverse and longitudinal shower profiles in ECAL and their specific ionisation in the TPC. A detailed description of the photon identification can be found in [14].

The iron return yoke is equipped with 23 layers of streamer tubes and forms the hadron calorimeter (HCAL). The latter provides a relative energy resolution of charged and neutral hadrons of $0.85 / \sqrt{E}$, with $E$ in $\mathrm{GeV}$. Muons are distinguished from hadrons by their distinct pattern in HCAL and by the muon chambers composed of two double-layers of streamer tubes outside HCAL.

The information from the tracking detectors and the calorimeters are combined in an energy flow algorithm [14. For each event, the algorithm provides a set of charged and neutral reconstructed particles, called energy flow objects, which are used in the analysis. Studies of $\mathrm{Z} \rightarrow \mathrm{q} \overline{\mathrm{q}}$ events show that the angular resolution of jets reconstructed from energy flow objects is typically $30 \mathrm{mrad}$ in space and the energy resolution approximately $\sigma_{E}=(0.6 \sqrt{E}+0.6)\left(1+\cos ^{2} \theta\right) \mathrm{GeV}$, where $E$ is the jet energy in $\mathrm{GeV}$ and $\theta$ is the polar angle with respect to the $\mathrm{z}$-axis along the $\mathrm{e}^{-}$beam direction.

\section{Monte Carlo generators}

Samples of fully simulated events, reconstructed with the same program as the data, are used for the design of the selections, determination of the signal efficiencies and the estimation of the background. The size of the generated signal samples correspond to 20 times (for the single- $\gamma$ and single- $\mathrm{W}$ analysis) and up to 80 times (for the $\mathrm{W}$-pair analysis) the collected 
luminosity.

The efficiency for the single- $\gamma$ cross section measurement is estimated using a modified version of the KORALZ 15] Monte Carlo program. The KORALZ generator simulates initial state photons using YFS exponentiation [16]. The generator is modified to include the effects from photons produced as bremsstrahlung off the exchanged virtual $\mathrm{W}$. This treatment includes the expected Standard Model contribution and possible anomalous couplings together with the interference of the two. The effect on the overall cross section is found to be small $(\sim 0.2 \%)$ for Standard Model couplings. However, it can be as large as a few percent in certain kinematical regions. The predictions of the modified KORALZ Monte Carlo are confirmed by an independent generator NUNUGPV [17], which is based on exact lowest order amplitudes for the production of up to three photons in the final state, modified for higher order QED effects using transverse momentum dependent structure functions.

For the single-W study the GRC4F program [18] is used to simulate the four-fermion signal process final state $e \nu f \bar{f}$. The effective QED coupling constant is fixed to be $\alpha_{\mathrm{QED}}=1 / 130.2$ as suggested in [19]. For initial state radiation, the photon structure function approach is utilised. Final state radiation and tau decays are simulated with PHOTOS [20] and TAUOLA [21].

For the analysis of W-pair final states, the KORALW [22] generator, which includes all four-fermion diagrams contributing to $\mathrm{W}^{+} \mathrm{W}^{-}$-like final states, is used to produce the primary reference sample with a $\mathrm{W}$ mass of $80.35 \mathrm{GeV} / \mathrm{c}^{2}$. The KORALW generator is interfaced with JETSET [23], PHOTOS [20], and TAUOLA [21] for fragmentation, final state radiation and $\tau$ decays, respectively. In addition, several samples are generated using the double resonant CC03 [9] diagrams with non-standard values for one coupling at a time, to check the reconstruction and TGC determination. Finally, a sample generated with the double resonant $\mathrm{CC} 03$ diagrams is used to optimise selection efficiencies and parametrise the corrections used in the kinematic fitting.

In order to include the effects from various background processes, Monte Carlo samples are generated with a corresponding integrated luminosity of each background sample of at least 20 times that of the data. PYTHIA [23] is used to generate $e^{+} e^{-} \rightarrow q \bar{q}(\gamma)$, ZZ, Zee, and $e^{+} e^{-} \rightarrow \mathrm{We} \nu$ event samples. In the ZZ sample, events with $\mathrm{W}^{+} \mathrm{W}^{-}$-like final states are discarded to avoid double counting. Two-photon processes are simulated with the PHOTO2 [24] generator. The KORALZ [15] and UNIBAB [25] generators are used for the di-lepton final states.

\section{Single- $\gamma$ production analysis}

Events with one or more photons and missing energy can be used to probe the anomalous WW $\gamma$ coupling parameters $\Delta \kappa_{\gamma}$ and $\lambda_{\gamma}$. Although the single- $\gamma$ channel is less sensitive to the couplings compared to the $\mathrm{W}$-pair and single- $\mathrm{W}$ channels, it provides complementary information. A detailed description of the Standard Model processes involved in the reaction $e^{+} e^{-} \rightarrow \nu \bar{\nu} \gamma(\gamma)$ and the modelling of the measured triple gauge-boson couplings can be found in $[$.

The sensitivity to the WW $\gamma$ couplings in the single- $\gamma$ channel comes from the $\mathrm{W}-\mathrm{W}$ fusion diagram. The W's exchanged in this t-channel diagram are predominantly at low momentum transfer. The single- $\gamma$ channel is therefore mainly sensitive to $\Delta \kappa_{\gamma}$ because contributions from $\lambda_{\gamma}$ contain higher powers of the $\mathrm{W}$ momenta. Furthermore, the effect of anomalous TGCs depends on the energy of the photon. For low energy photons, below 
the radiative return to the $\mathrm{Z}$ peak the sensitivity arises from the interference between the Standard Model and the anomalous contribution; this interference has a linear dependence on the TGCs. In the region around the radiative return to the $\mathrm{Z}$ peak, the sensitivity is minimal. For high energy photons, above the radiative return to the $\mathrm{Z}$ peak, the dependence on the TGCs is quadratic.

\subsection{Event selection and determination of the TGCs}

The events are selected from the $189 \mathrm{GeV}$ data sample using the procedure described in [26]. In summary, single- $\gamma$ events are selected by requiring at least one photon candidate with $\theta_{\gamma}>20^{\circ}$ and $p_{\mathrm{T}}^{\gamma} / E_{\text {beam }}>0.1$ and no additional activity in form of reconstructed charged tracks or energy deposits in the forward regions (below $14^{\circ}$ ). Events where a photon has converted into a $e^{+} e^{-}$pair are not considered.

Anomalous contributions to the WW $\gamma$ vertex increase the total cross section and lead to characteristic energy and angular distributions of the final state photons. For the single$\gamma$ channel the TGCs are extracted from the data by performing a maximum likelihood fit based on the overall number of observed photons, their polar angles $\theta_{\gamma}$ and scaled energies $x_{E}\left(=E_{\gamma} / E_{\text {beam }}\right)$ of the form

$$
\log L=\log \frac{\left(N_{\text {exp }}\right)^{N_{\text {obs }}} \mathrm{e}^{-N_{\text {exp }}}}{N_{\text {obs }} !}+\sum \log P_{i},
$$

where $P_{i}$ is the probability density function of observing event $i$ with a given value of $x_{E}$ and $\theta_{\gamma}$ and $N_{\exp }$ is the expected number of events including background. The probability density function and the expected number of events for different values of the couplings are constructed by reweighting fully simulated single- $\gamma$ events. Distributions of the polar angle and the scaled energy for single- $\gamma$ events are shown in Figure 11.

Two separate kinematic regions are used in the fit, excluding a region around the $\mathrm{Z}$ peak return, where the sensitivity is small. Defining $E_{\gamma}^{\mathrm{Z}}=\left(s-m_{\mathrm{Z}}^{2}\right) /(2 \sqrt{s})$, the excluded region is $E_{\gamma}^{\mathrm{Z}}-3 \Gamma_{\mathrm{Z}}<E_{\gamma}<E_{\gamma}^{\mathrm{Z}}+0.5 \Gamma_{\mathrm{Z}}$. The total numbers of photons used in the fit are 120 (128 expected) and 260 (258 expected) below and above the excluded region.

\subsection{Results}

At present energies, the cross section and the shape contribute equally in the likelihood function for $\Delta \kappa_{\gamma}$, whereas the result for $\lambda_{\gamma}$ is dominated by the sensitivity to the shape above the excluded region. The estimation of the systematic uncertainties follows the procedure described in Ref. [4] and the different contributions are summarised in Table 11. The fitted results for the $189 \mathrm{GeV}$ data for single parameter fits, where each coupling is determined setting the other coupling to its Standard Model value, are

$$
\begin{aligned}
\Delta \kappa_{\gamma} & =0.4 \pm 0.7 \pm 0.2 & & \left(\lambda_{\gamma}=0\right) \\
\lambda_{\gamma} & =0.3 \pm 0.9 \pm 0.2 & & \left(\Delta \kappa_{\gamma}=0\right)
\end{aligned}
$$

where the first error is the statistical error and the second is the systematic uncertainty. The 95\% confidence level limits including systematic errors are:

$$
\begin{array}{lll}
-1.1<\Delta \kappa_{\gamma}<1.8 & \left(\lambda_{\gamma}=0\right) \\
-1.5<\lambda_{\gamma}<2.0 & \left(\Delta \kappa_{\gamma}=0\right) .
\end{array}
$$


Table 1: Summary of the systematic errors on single parameter fits for $\Delta \kappa_{\gamma}$ and $\lambda_{\gamma}$ from the single- $\gamma$ analysis at $189 \mathrm{GeV}$.

\begin{tabular}{|l|c|c|}
\hline Source & $\Delta \kappa_{\gamma}$ & $\lambda_{\gamma}$ \\
\hline \hline Acceptance corrections & 0.08 & 0.08 \\
Photon energy calibration & 0.11 & 0.14 \\
Background & 0.05 & 0.05 \\
Luminosity & 0.03 & 0.03 \\
Theoretical uncertainty & 0.13 & 0.15 \\
\hline Total & 0.20 & 0.22 \\
\hline
\end{tabular}

The validity of these $95 \%$ C.L. limits and the error from the likelihood fit have been checked using many Monte Carlo samples corresponding to the data luminosity as described in [4.

Combining with the previous measurement for centre-of-mass energies between 161 and $183 \mathrm{GeV}$ [4], the $95 \%$ C.L. limits on $\Delta \kappa_{\gamma}$ and $\lambda_{\gamma}$ from single- $\gamma$ production are

$$
\begin{aligned}
& -1.0<\Delta \kappa_{\gamma}<1.5 \quad\left(\lambda_{\gamma}=0\right), \\
& -1.4<\lambda_{\gamma}<1.8 \quad\left(\Delta \kappa_{\gamma}=0\right) .
\end{aligned}
$$

The negative log-likelihood functions curves are shown in Figure 2 for the $189 \mathrm{GeV}$ data, 161 - $183 \mathrm{GeV}$ data [1] and the combined results. In the combination, the systematic errors from acceptance and theoretical prediction are assumed to be fully correlated, while all other sources are taken as uncorrelated.

\section{Single-W production analysis}

Single-W production, $e^{+} e^{-} \rightarrow \mathrm{We} \nu$, is sensitive to the WW $\gamma$ vertex. This sensitivity comes from the $\gamma-\mathrm{W}$ fusion diagram, where the momentum transfer is low. As for the single- $\gamma$ channel, the single-W channel is therefore mostly sensitive to $\Delta \kappa_{\gamma}[27]$.

\subsection{Selection}

The analysis of single-W production is performed on the $189 \mathrm{GeV}$ data sample. All W decay modes are used and the selection of each W decay final state, described in the following, has been optimised for the single-W signal definition used in the previous analysis at lower centre-of-mass energies [3]:

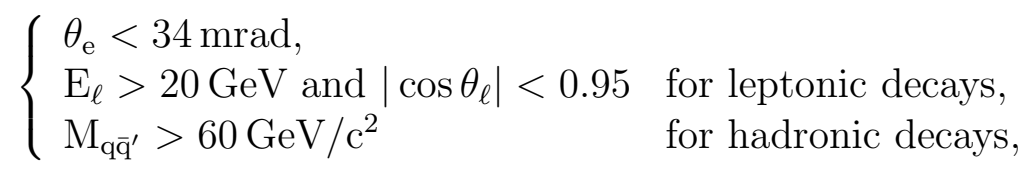

where $\theta_{\mathrm{e}}$ is the polar angle of the scattered electron, $\mathrm{E}_{\ell}$ and $\theta_{\ell}$ are the energy and polar angle of leptons from the $\mathrm{W}$ decay. $\mathrm{M}_{\mathrm{q} \overline{\mathrm{q}}^{\prime}}$ is the invariant mass of the quark pair. The cut angle at $34 \mathrm{mrad}$ corresponds to the lower edge of the acceptance of the ALEPH detector.

As single-W production is dominated by t-channel processes, the outgoing electron is predominantly emitted at small polar angles. Another specific feature is the large missing momentum carried away by the electron-neutrino, and therefore a common selection criterium for all single- $W$ final states is the requirement of the missing momentum direction to be within the detector acceptance, $\left|\cos \theta_{\text {miss }}\right|<0.9$. 


\subsubsection{Leptonic Selection}

The leptonic W decay is characterised by a high energy isolated lepton. Allowing for a multiprong decay of the tau, events with one or three good tracks $(|\cos \theta|<0.95)$ are accepted. The selection cuts are the same as in the analysis in Ref. [3] and are summarised here.

In addition to the cut on the missing momentum direction, tagged two-photon events are rejected by requiring that no energy be detected within a cone of $12^{\circ}$ around the beam axis $\left(\mathrm{E}_{12}=0\right)$.

The remaining backgrounds, mainly untagged two-photon events and two-fermion events, are eliminated by requiring that the transverse missing momentum be greater than $0.06 \sqrt{s}$. This threshold is increased to $0.1 \sqrt{s}$ if the missing momentum direction points to within $10^{\circ}$ in azimuth to the boundaries between the two LCAL halves or between the six inner sectors for the TPC. It is required that no energy is found within a wedge of $10^{\circ}$ opposite to the direction of the lepton transverse momentum. To reduce the background from $e^{+} e^{-} \rightarrow$ Zee with $\mathrm{Z}$ decaying to neutrinos, events are rejected if an electron candidate track is identified and its energy, including the neutral energy in a $10^{\circ}$ cone around the track, is less than $20 \mathrm{GeV}$.

The selection efficiencies for the three final states are $75 \%(e \nu e \nu), 77 \%(e \nu \mu \nu)$ and $43 \%$ $(e \nu \tau \nu)$. The main background source is Zee where the Z decays to $\nu_{\mu} \bar{\nu}_{\mu}$ or $\nu_{\tau} \bar{\nu}_{\tau}$ (the $\nu_{\mathrm{e}} \bar{\nu}_{\mathrm{e}}$ case is a four-fermion final state which is $e^{+} e^{-} \rightarrow \mathrm{We \nu}$ like and is part of the signal).

In the data, 23 events are observed in agreement with the expectation from the Standard Model of 26.5 events (17.7 signal events). The composition is 15 events with an electron (8.4 signal and 6.2 background expected), 4 events with a muon (6.6 signal and 0.4 background expected) and 4 events with a tau (2.7 signal and 2.2 background expected). The distributions of the lepton transverse momentum, $p_{\mathrm{T}}^{l}$, for single-W events passing the final selection cuts for the leptonic electron and muon $\mathrm{W}$ decay, are shown in Figure 3 .

\subsubsection{Hadronic selection}

For the hadronic W decay, the event topology is characterised by two acoplanar jets with an invariant mass around that of the $\mathrm{W}$ boson. The selection is the same as in the analysis in Ref. [3]. In addition to the cut on missing momentum direction, at least seven good tracks are required. Similarly to the leptonic selection, tagged two-photon events and two-fermion events with initial state radiation are rejected by demanding that the energy $\mathrm{E}_{12}$ be less than $0.025 \sqrt{s}$. The visible mass is required to exceed $60 \mathrm{GeV} / \mathrm{c}^{2}$ and to be less than $90 \mathrm{GeV} / \mathrm{c}^{2}$ to reject untagged two-photon events at the low end of the mass spectrum and ZZ events at the high end.

Events for which the energy in a wedge of $30^{\circ}$ centred on the transverse missing momentum direction is greater than $0.1 \sqrt{s}$ are rejected. The acollinearity angle between the two hemisphere (defined by the event thrust axis) momentum directions is required to be less than $165^{\circ}$.

The semileptonic final state $(\ell \nu q \bar{q})$ of $\mathrm{W}$-pair production is efficiently rejected by requiring that no identified electron or muon with an energy of more than $0.05 \sqrt{s}$ be reconstructed. The tau jet reconstruction algorithm of Ref. [3] is used in order to further reject semileptonic decays of W-pairs which contain a tau lepton. After all cuts, the semileptonic W-pair production, primarily events with one tau lepton, remains as the dominant background.

The efficiency for the hadronic $\mathrm{W}$ channel is about $43 \%$. In the data, 53 events are observed, in agreement with the Standard Model expectation of 63.1 events (23.5 signal 
Table 2: Summary of the relative systematic uncertainties in the expected numbers of selected signal events of the leptonic and the hadronic channels from the single- $W$ analysis at $189 \mathrm{GeV}$.

\begin{tabular}{|l|c|c|}
\hline Source & $\Delta N_{\text {lep }}^{\text {exp }} / N_{\text {lep }}^{\text {exp }}$ & $\Delta N_{\text {had }}^{\text {exp }} / N_{\text {had }}^{\text {exp }}$ \\
\hline \hline Luminosity & \pm 0.01 & \pm 0.03 \\
Calorimeter calibration & - & ${ }_{-0.08}^{+0.11}$ \\
E $_{12}$ inefficiency & \pm 0.01 & - \\
Signal and background cross-section & \pm 0.06 & \pm 0.05 \\
Fragmentation & - & \pm 0.05 \\
\hline Total & \pm 0.07 & ${ }_{-0.11}^{+0.13}$ \\
\hline
\end{tabular}

events). The visible mass distribution of the selected events is displayed in Figure 3 .

\subsection{Results}

Limits on $\Delta \kappa_{\gamma}$ and $\lambda_{\gamma}$ are derived from the total rate of single-W events, which is sensitive to the WW $\gamma$ couplings. The upper limit on the single-W signal cross section has been calculated while varying only one coupling at a time, and the $95 \%$ C.L. limits on $\Delta \kappa_{\gamma}$ and $\lambda_{\gamma}$ for the $189 \mathrm{GeV}$ data are

$$
\begin{aligned}
& -2.09<\Delta \kappa_{\gamma}<0.20 \quad\left(\lambda_{\gamma}=0\right) \\
& -0.77<\lambda_{\gamma}<0.79 \quad\left(\Delta \kappa_{\gamma}=0\right),
\end{aligned}
$$

including the systematic uncertainties. The different contributions to the systematic errors are summarised in Table 2. The total systematic error amounts to $7 \%$ for the leptonic and $12 \%$ for the hadronic channel on the predicted numbers of signal events. The overall systematic errors are small compared to the statistical precision, which amounts to $33 \%$ for the leptonic channel and $55 \%$ for the hadronic channel.

Combining with the previous measurement for centre-of-mass energies between 161 and $183 \mathrm{GeV}$ [3], the $95 \%$ C.L. limits on $\Delta \kappa_{\gamma}$ and $\lambda_{\gamma}$ from single-W are

$$
\begin{aligned}
& -2.12<\Delta \kappa_{\gamma}<0.23 \quad\left(\lambda_{\gamma}=0\right), \\
& -0.76<\lambda_{\gamma}<0.78 \quad\left(\Delta \kappa_{\gamma}=0\right) .
\end{aligned}
$$

The corresponding $\log L$ curves are shown in Figure 4 for $\Delta \kappa_{\gamma}\left(\lambda_{\gamma}=0\right)$ and $\lambda_{\gamma}\left(\Delta \kappa_{\gamma}=0\right)$ for the $189 \mathrm{GeV}$ data, 161 - $183 \mathrm{GeV}$ data and the combined results.

\section{W-Pair production analysis}

The large number of W-pair events produced yield the dominant sensitivity to the TGCs. The process is sensitive to both the $\mathrm{WW} \gamma$ and the WWZ couplings via the s-channel $\mathrm{W}$ pair production diagrams and the sensitivity to the coupling $\lambda_{\gamma}$ is higher than that of the single- $\mathrm{W}$ and the single- $\gamma$ processes.

\subsection{Event selection and kinematic reconstruction}

In this section the event selections for the three distinct $\mathrm{W}^{+} \mathrm{W}^{-}$event topologies, $\ell \nu q \bar{q}$, $q \bar{q} q \bar{q}$, and $\ell \nu \ell \nu$, are described. Selected events are exclusively classified in the following 
Table 3: The numbers of events after all cuts applied in the final $\mathrm{W}^{+} \mathrm{W}^{-}$TGC results for data and Monte Carlo simulation in all channels at centre-of-mass energies of 183 and $189 \mathrm{GeV}$. The number of Monte Carlo events is normalised to the respective integrated luminosity of the data. The quoted efficiencies $\epsilon$ and purities $p$ are determined from $\mathrm{CC} 03$ events with $m_{\mathrm{W}}=80.35 \mathrm{GeV} / \mathrm{c}^{2}$. For a given $\mathrm{W}^{+} \mathrm{W}^{-}$channel, contributions from other channels are considered as background.

\begin{tabular}{|l|c|c|c|c|c||c|c|c|c|c|}
\hline & \multicolumn{5}{|c||}{$\sqrt{s}=183 \mathrm{GeV}$} & \multicolumn{5}{c|}{$\sqrt{s}=189 \mathrm{GeV}$} \\
\hline & $e \nu q \bar{q}$ & $\mu \nu q \bar{q}$ & $\tau \nu q \bar{q}$ & $q \bar{q} q \bar{q}$ & $\ell \nu \ell \nu$ & $e \nu q \bar{q}$ & $\mu \nu q \bar{q}$ & $\tau \nu q \bar{q}$ & $q \bar{q} q \bar{q}$ & $\ell \nu \ell \nu$ \\
\hline \hline $\mathrm{N}_{\exp }$ & 105.6 & 107.1 & 90.5 & 296.8 & 29.7 & 360.6 & 369.9 & 230.2 & 1202.9 & 101.3 \\
$\mathrm{~N}_{\text {data }}$ & 117 & 95 & 88 & 314 & 29 & 361 & 370 & 224 & 1130 & 102 \\
\hline \hline$\epsilon(\%)$ & 76.2 & 79.5 & 50.7 & 67.0 & 61.9 & 74.0 & 78.1 & 44.8 & 78.6 & 61.9 \\
$p(\%)$ & 96.3 & 97.8 & 73.5 & 90.0 & 89.8 & 96.7 & 98.1 & 80.6 & 86.2 & 90.3 \\
\hline
\end{tabular}

order of priority: $\mu \nu q \bar{q}, e \nu q \bar{q}, q \bar{q} q \bar{q}, \tau \nu q \bar{q}$, and $\ell \nu \ell \nu$. The expected numbers of events after all cuts used in the TGC results for signal and background processes at both centre-of-mass energies are summarised in Table 3 for each channel, along with the corresponding selection efficiencies and purities.

\subsection{1 $\mathrm{W}^{+} \mathrm{W}^{-} \rightarrow e \nu q \bar{q}$ and $\mathrm{W}^{+} \mathrm{W}^{-} \rightarrow \mu \nu q \bar{q}$ events}

The event selection procedure for semileptonic $\mathrm{W}^{+} \mathrm{W}^{-}$events is similar to that used for the $\mathrm{W}$ mass measurement at the corresponding energy [28,29]. At $183 \mathrm{GeV}$, events are reconstructed such that they contain a high energy lepton candidate and two jets [28]. The charged particle with the highest momentum component anti-parallel to the missing momentum is chosen as lepton candidate. At $189 \mathrm{GeV}$ the selection criteria for the lepton track are slightly changed, using the lepton track isolation [22]. The DURHAM-PE [28] clustering algorithm is applied to all energy flow objects not used to construct the lepton four-momentum, and these are forced into two jets. After this preselection, the probability for the event being signal is determined using the momentum of the lepton, the total missing transverse momentum and the lepton isolation from the closest jet.

At this stage events passing a cut on the probability are subjected to a kinematic fit in order to improve the resolution on the reconstructed four-momenta of the $\mathrm{W}$ decay products. The kinematic fit and additional reconstruction cuts are described in the context of the specific TGC analysis.

A W-pair event can be characterised by the five measured angles, $\theta_{W}$, the $\mathrm{W}^{-}$production angle between the $\mathrm{W}^{-}$and initial $e^{-}$in the $\mathrm{W}^{+} \mathrm{W}^{-}$rest frame, the polar and azimuthal angles of the lepton, $\theta_{1}^{*}$ and $\phi_{1}^{*}$, in the rest frame of its parent $\mathrm{W}$ and the polar and azimuthal angles of a quark jet, $\theta_{\text {jet }}^{*}$ and $\phi^{*}$ jet, in the rest frame of its parent $\mathrm{W}$. The distributions of the five angles $\cos \theta_{W}, \cos \theta_{l}^{*}, \phi_{l}^{*}, \cos \theta_{\text {jet }}^{*}$ and $\phi_{\text {jet }}^{*}$, for $e \nu q \bar{q}$ and $\mu \nu q \bar{q}$ events at $189 \mathrm{GeV}$ after selection and reconstruction is represented in Figure 5 .

\subsection{2 $\mathrm{W}^{+} \mathrm{W}^{-} \rightarrow \tau \nu q \bar{q}$ events}

The event selection is based on two complementary algorithms, developed for the cross section measurement at 161 [30] and $172 \mathrm{GeV}$ [31], but modified to account for the change in event kinematics with centre-of-mass energy. In summary, events passing a set of preselection cuts are selected as semileptonic $\tau$ candidate events if they fulfil either a global or topological 
selection. The DURHAM-PE clustering algorithm is then applied to all energy flow objects that are not used to construct the tau four-momentum, and these are forced into two jets. More detailed descriptions of the selection and tau reconstruction can be found in the publications on the $\mathrm{W}$ mass determination at the corresponding energies [28, 29].

To improve the resolution of the angular observables a 3 -constraint kinematic fit is applied, requiring four-momentum conservation and reference mass constraints. In the kinematic fit the direction of the $\tau$ is approximated by its visible decay products and the extra energy loss is compensated by correction coefficients obtained from Monte Carlo simulated $\mathrm{W}^{+} \mathrm{W}^{-} \rightarrow \tau \nu q \bar{q}$ events. For single prong $\tau$ decays the charge of the $\tau$ is directly accessible, but in the case of three-prong $\tau$ decays ambiguities arise due to mis-assigned particles from the jets to the $\tau$. For three-prong $\tau$ decays the charge of the $\tau$ is therefore determined from the sign of the pseudorapidity-weighted average jet charge of the $\tau$ decay products (Section 6.1.3), where the pseudo-rapidity is defined with respect to jet-axis. The charge mis-assignment in $\tau \nu q \bar{q}$ events is $5 \%$ for one-prong and $41 \%$ for three-prong $\tau$ decays.

The distribution of the cosine of the $\mathrm{W}^{-}$production angle from $\tau \nu q \bar{q}$ events can be seen in Figure 6 .

\subsection{3 $\mathrm{W}^{+} \mathrm{W}^{-} \rightarrow q \bar{q} q \bar{q}$ events}

To extract the hadronic $\mathrm{W}^{+} \mathrm{W}^{-}$signal with high purity and efficiency, the selection is based on a neural network 32. Events passing a preselection designed to remove the q $\bar{q}(\gamma)$ background, are assigned a neural network output, based on global event properties, heavy quark flavour tagging, jet properties and WW kinematics. A detailed description of the selection algorithm at 183 and $189 \mathrm{GeV}$ is given in 32.

For the hadronic $\mathrm{W}^{+} \mathrm{W}^{-}$events the reconstruction of the relevant information is more complicated since the $\mathrm{W}^{-}$direction is not known and the information on the particle flavours in either $\mathrm{W}$ system is not discriminant. In this case the four jets can be paired in three different ways. To select the best pairing, a 6-constraint kinematic fit is applied to all three possible pairings. The kinematic fit requires four-momentum conservation and reference mass constraints. The four-momenta obtained in the kinematic fit for the pairing with the lowest $\chi^{2}$ value are then used in the final determination of the TGCs, while the other combinations are discarded. The efficiency of this algorithm to find the correct combination was found to be $78 \%$ at 183 and $75 \%$ at $189 \mathrm{GeV}$.

To assign a jet pair to the $\mathrm{W}^{+}$or $\mathrm{W}^{-}$a jet charge algorithm is used. The jet charge, $Q_{\text {jet }}$, is obtained from the pseudorapidity-weighted average charge of jet particles. The jet pair charge is defined by the sum of the two jets assigned to a $\mathrm{W}, Q_{\mathrm{W}}=Q_{\text {jet1 }}+Q_{\text {jet2}}$. A jet pair is then assigned to the $\mathrm{W}^{+}$based on the charge difference between the two jet pairs, $\Delta Q$, with a probability $P_{+}$. The probability $P_{+}$is given by

$$
P_{+}(\Delta Q)=\frac{\mathcal{N}_{+}(\Delta Q)}{\mathcal{N}_{+}(\Delta Q)+\mathcal{N}_{+}(-\Delta Q)},
$$

where $\mathcal{N}_{+}$is the probability density function for the charge difference between the two $\mathrm{W}$ systems for true $\mathrm{W}^{+}$jet pairs obtained from Monte Carlo event samples [1]. Figure 7 shows the distribution of the di-jet charge of the two $\mathrm{W}$ systems for true $\mathrm{W}^{+}$and $\mathrm{W}^{-}$decays, obtained from Monte Carlo generator information. The distribution of the di-jet charge from semileptonic events is also shown for both data and Monte Carlo. The data are well reproduced by the Monte Carlo simulation. The charge assignment efficiency for correctly paired hadronic $\mathrm{W}^{+} \mathrm{W}^{-}$events amounts to approximately $76 \%$ for $P_{+}>0.5$. 
The distribution of the cosine of the $\mathrm{W}^{-}$production angle from $q \bar{q} q \bar{q}$ events can be found in Figure 6.

\subsection{4 $\mathrm{W}^{+} \mathrm{W}^{-} \rightarrow \ell \nu \ell \nu$ events}

The selection of $\mathrm{W}^{+} \mathrm{W}^{-} \rightarrow \ell \nu \ell \nu$ events (where $\ell$ denotes an electron or muon) is mainly based on variables used in Ref. [30], namely missing transverse momentum, missing mass and kinematic properties of the lepton candidate. The information of these and other variables is combined in a neural network. A detailed description of all the variables used in the neural network can be found in the appendix A.

For purely leptonic $\mathrm{W}^{+} \mathrm{W}^{-}$events the momenta of the two neutrinos are unknown. However, in the absence of ISR and neglecting the $W$ width, the constraint that the two $l \nu$ systems should have the $\mathrm{W}$ mass $\left(M_{1}=M_{2}=80.35 \mathrm{GeV} / c^{2}\right)$ in combination with the usual four-momentum conservation allows a reconstruction of the neutrino momenta. The quadratic nature of the mass constraint results in a two-fold ambiguity, corresponding to flipping both neutrinos with respect to the plane defined by the charged leptons. As detector resolution, ISR and the finite $\mathrm{W}$ width are not included in this reconstruction hypothesis, $28.0 \%$ of the events have no physical solution and a zero-constrained kinematic fit is employed. The fit determines a set of values for the reference masses, $M_{1}$ and $M_{2}$, in the mass-constraints for the two $l \nu$ systems, for which a physical solution exists. By this method $92 \%$ of the events without a solution are recovered, resulting in a $97.7 \%$ reconstruction efficiency for signal events. The majority of the events which fail to have a solution are purely leptonic $\mathrm{W}^{+} \mathrm{W}^{-}$events with at least one leptonically decaying $\tau$, which is the dominant background. In addition to the selection by a cut on the neural network output, purely leptonic events are only accepted if a physical solution is found inside the mass window of $55-105 \mathrm{GeV} / c^{2}$.

The distribution of the cosine of the $\mathrm{W}^{-}$production angle for $\ell \nu \ell \nu$ events at $189 \mathrm{GeV}$ after selection and reconstruction is represented in Figure 6 .

\subsection{Determination of the TGCs}

Three different methods, described in the following, are used to extract the couplings in the different W-pair final states.

\subsubsection{The optimal observable methods}

The general idea of optimal observables (OO) [33] is to project the sensitive kinematic information for a given coupling $g_{i}$ onto the one-dimensional distribution of a suitably defined variable $\mathcal{O}_{i}^{(1)}$. The coupling $g_{i}$ can then be extracted from a fit to this distribution or, equivalently, from the measurement of the mean value $\left\langle\mathcal{O}_{i}^{(1)}\right\rangle$ of the optimal observable.

Since the amplitudes are linear in the TGCs the differential cross section can be expanded in these couplings $g_{i}$ containing no terms beyond the second order

$$
\frac{d \sigma}{d \Omega}=S_{0}(\Omega)\left(1+\sum_{i} \mathcal{O}_{i}^{(1)} \cdot g_{i}+\sum_{i j} \mathcal{O}_{i j}^{(2)} \cdot g_{i} g_{j}\right),
$$

where $g_{i}$ denotes any type of couplings and $\Omega$ denotes phase space variables taking into account reconstruction ambiguities for the individual $\mathrm{W}^{+} \mathrm{W}^{-}$channels. The zero-order term, 
$S_{0}(\Omega)$, represents the Standard Model contribution. Using the first order term, a given set of couplings, $\bar{g}$, can be determined by minimising

$$
\chi^{2}(\bar{g})=\sum_{i j}\left(\left\langle\mathcal{O}_{i}^{(1)}\right\rangle-E\left[\mathcal{O}_{i}^{(1)}\right]\right) V(\mathcal{O})_{i j}^{-1}\left(\left\langle\mathcal{O}_{j}^{(1)}\right\rangle-E\left[\mathcal{O}_{j}^{(1)}\right]\right)
$$

where $\left\langle\mathcal{O}_{i}^{(1)}\right\rangle$ and $V(\mathcal{O})$ are the measured mean values and their covariance matrix. The expected mean values, $E\left[\mathcal{O}_{i}^{(1)}\right]$, are obtained by reweighting of fully simulated $\mathrm{W}^{+} \mathrm{W}^{-}$events.

In order to ensure maximal sensitivity, $g_{i}$ can be determined by two different approaches:

- An iterative procedure (denoted $\mathbf{O O}_{1}$ in the further text), where the cross section in Eq. 3 is expanded about a given coupling value and consequently the definition of the observable $\mathcal{O}_{i}^{(1)}$ is re-optimised.

- Adding the information contained in the second order term of the expansion in Eq. 3 (called $\mathbf{O O}_{2}$ in the further text). This is achieved by including the second optimal observable and adding additional terms of the same structure as the ones in Eq. 4 to the $\chi^{2}$, including new terms describing the correlation between $\mathcal{O}_{i}^{(1)}$ and $\mathcal{O}_{i j}^{(2)}$. The second order observable increases the sensitivity when the information contained in the first order observable decreases [34]. The covariance matrix for the mean values are obtained by reweighting fully simulated $\mathrm{W}^{+} \mathrm{W}^{-}$events.

In both cases the information from the measured cross section is included by adding a Poisson term to the likelihood function.

By construction, these methods are bias-free and take into account any experimental effect, provided that the Monte Carlo simulation describes the data correctly. For a given channel, contributions from any other channel are considered as coupling dependent backgrounds. In addition the efficiencies and purities of each selection are parameterised as function of the couplings.

For semileptonic events, both $\mathrm{OO}$ analyses, $\mathrm{OO}_{1}$ and $\mathrm{OO}_{2}$, apply a two-constraint kinematic fit using four-momentum conservation, equal W-mass hypothesis and including a massless neutrino. The corresponding $\chi^{2}$-probability of the fit is required to be larger than 0.005 for an event to be selected for the TGC extraction. This cut improves the purity of the sample and discards poorly reconstructed events. In addition, an OO-window cut is applied, optimised to improve the sensitivity to the TGCs by reducing the contamination of background events with $\mathrm{OO}$ values incompatible with $\mathrm{W}$-pair production.

For hadronically decaying W's, there remains a twofold ambiguity since the quark flavours are undetermined. Hence, for semileptonic $e \nu q \bar{q}, \mu \nu q \bar{q}$ and $\tau \nu q \bar{q}$ events the contributions are averaged over the quark and anti-quark directions. For hadronic events, considering the $\mathrm{W}$ charge as undetermined, there is an eightfold ambiguity. This is included in the final extraction of the TGCs, where each contribution is weighted with the corresponding di-jet charge probability, Eq. 2. For leptonic events, $\ell \nu \ell \nu$, the contributions are averaged over the two solutions for the neutrino momenta.

\subsubsection{Maximum likelihood-fit}

A maximum likelihood analysis (LL), Eq. 1, of the channels $e \nu q \bar{q}$ and $\mu \nu q \bar{q}$ is used to measure the $\mathrm{C}$ - or P-violating couplings and as a cross-check for the CP-conserving couplings. The measured variables are the five angles described in Sect. 6.1.1. As in the optimal observable 
Table 4: The numbers of events after all cuts applied in the maximum likelihood TGC analysis for data and Monte Carlo simulation at centre-of-mass energies of 183 and $189 \mathrm{GeV}$. The number of Monte Carlo events is normalised to the respective integrated luminosity of the data. The quoted efficiencies $\epsilon$ and purities $p$ are determined from $\mathrm{CC} 03$ events with $m_{\mathrm{W}}=80.35 \mathrm{GeV} / \mathrm{c}^{2}$. Only non- $\mathrm{W}^{+} \mathrm{W}^{-}$events are considered as background in the calculation of the efficiencies $\epsilon$ and purities $p$.

\begin{tabular}{|l|c|c||c|c|}
\hline & \multicolumn{2}{|c||}{$\sqrt{s}=183 \mathrm{GeV}$} & \multicolumn{2}{c|}{$\sqrt{s}=189 \mathrm{GeV}$} \\
\hline Channel & $e \nu q \bar{q}$ & $\mu \nu q \bar{q}$ & $e \nu q \bar{q}$ & $\mu \nu q \bar{q}$ \\
\hline \hline $\mathrm{N}_{\exp }$ & 91.8 & 97.4 & 293.0 & 311.7 \\
$\mathrm{~N}_{\text {data }}$ & 98 & 86 & 275 & 310 \\
\hline \hline$\epsilon(\%)$ & 66.4 & 71.0 & 66.1 & 70.9 \\
$p(\%)$ & 98.5 & 99.4 & 98.6 & 99.5 \\
\hline
\end{tabular}

analyses, no quark flavour tagging is performed and the quark and anti-quark directions are averaged. The probability density function, $P$, is given by

$$
P(\bar{\Omega}, \bar{g})=\frac{b(\bar{\Omega})+s(\bar{\Omega}, \bar{g})}{B+S(\bar{g})}
$$

where $\bar{g}$ denotes a set of couplings and the angles $\bar{\Omega}=\left(\theta_{W}, \theta_{1}^{*}, \phi_{1}^{*}, \theta_{\text {jet }}^{*}, \phi_{\text {jet }}^{*}\right)$ are calculated using the charged lepton, neutrino, and quark jet four-vectors. The quantity $b(\bar{\Omega})$ is the background distribution as predicted by Monte Carlo. The $\mathrm{W}^{+} \mathrm{W}^{-}$signal distribution, $s(\bar{\Omega}, \bar{g})$, is defined by

$$
s(\bar{\Omega}, \bar{g})=\int d \hat{s} d \bar{\Omega}^{\text {true }} r\left(\bar{\Omega}, \bar{\Omega}^{\text {true }}\right) \epsilon\left(\hat{s}, \bar{\Omega}^{\text {true }}\right) F(\hat{s}) \frac{d \sigma}{d \bar{\Omega}^{\prime}}\left(\hat{s}, \bar{\Omega}^{\text {true }}, \bar{g}\right),
$$

where $\hat{s}$ is the squared invariant mass of the $W^{+} W^{-}$system, $r\left(\bar{\Omega}, \bar{\Omega}^{\text {true }}\right)$ is the detector resolution function, $\epsilon\left(\hat{s}, \bar{\Omega}^{\text {true }}\right)$ is the detection efficiency, $F(\hat{s})$ is an initial state radiation function [35], and $\frac{d \sigma}{d \Omega^{\prime}}\left(\hat{s}, \bar{\Omega}^{\text {true }}, \bar{g}\right)$ is the lowest-order narrow-width differential cross-section for $\mathrm{W}^{+} \mathrm{W}^{-}$production and decay [7].

The normalisation factors $B$ and $S(\bar{g})$ are the integrals of $b(\bar{\Omega})$ and $s(\bar{\Omega}, \bar{g})$, where $S(\bar{g})$ is evaluated by reweighting $W^{+} W^{-}$Monte Carlo events in order to include detector resolution and efficiency. The proper evaluation of the normalisation $S(\bar{g})$ is crucial for the success of the likelihood method.

Some approximations to $s(\bar{\Omega}, \bar{g})$ are made when evaluating the numerator of Eq. 5. In particular, most of the information in the detection efficiency function $\epsilon(\hat{s}, \bar{\Omega})$ arises from the charged lepton momentum $p_{l}$ and polar angle $\theta_{l}$ in the laboratory reference frame. This dependence has been parameterised with a two-dimensional efficiency function.

The event selection for this method is the same as for the optimal observables. A kinematic fit using the equal mass hypothesis and four-momentum conservation is applied, where the corresponding $\chi^{2}$-probability is required to be larger than 0.02 . The efficiencies, purities and numbers of events for data and Monte Carlo simulation are shown in Table 4 .

\subsection{Results}

\subsubsection{Comparison of methods}

The three methods $\left(\mathrm{OO}_{1}, \mathrm{OO}_{2}\right.$ and the maximum likelihood) discussed above are used to determine the TGCs from WW events. 
Table 5: Comparison of the three couplings $\Delta \mathrm{g}_{1}^{\mathrm{Z}}, \Delta \kappa_{\gamma}$ and $\lambda_{\gamma}$, using the three methods $\mathrm{OO}_{1}, \mathrm{OO}_{2}$ and maximum likelihood. The error intervals for each coupling are statistical only.

\begin{tabular}{|c|c|c|c|c|}
\hline \multirow[b]{2}{*}{ Channel } & \multirow[b]{2}{*}{ Coupling } & \multicolumn{3}{|c|}{ Method } \\
\hline & & $\mathrm{OO}_{1}$ & $\mathrm{OO}_{2}$ & LL \\
\hline \multirow{3}{*}{$e \nu q \bar{q}$} & $\Delta \mathrm{g}_{1}^{\mathrm{Z}}$ & $0.07_{-0.10}^{+0.11}$ & $0.10_{-0.10}^{+0.09}$ & $0.03_{-0.10}^{+0.10}$ \\
\hline & $\Delta \kappa_{\gamma}$ & $-0.09_{-0.34}^{+0.50}$ & $0.45_{-0.33}^{+0.35}$ & $0.11_{-0.30}^{+0.47}$ \\
\hline & $\lambda_{\gamma}$ & $0.04_{-0.11}^{+0.12}$ & $0.22_{-0.10}^{+0.11}$ & $0.11_{-0.11}^{+0.11}$ \\
\hline \multirow{3}{*}{$\mu \nu q \bar{q}$} & $\Delta g_{1}^{Z}$ & $0.05_{-0.10}^{+0.10}$ & $0.00_{-0.08}^{+0.10}$ & $0.06_{-0.09}^{+0.09}$ \\
\hline & $\Delta \kappa_{\gamma}$ & $-0.02+0.52$ & $0.24_{-0.35}^{+0.59}$ & $0.38_{-0.38}^{+0.49}$ \\
\hline & $\lambda_{\gamma}$ & $-0.03_{-0.09}^{+0.10}$ & $-0.08_{-0.08}^{+0.09}$ & $-0.08+0.09$ \\
\hline \multirow{3}{*}{$\tau \nu q \bar{q}$} & $\Delta g_{1}^{Z}$ & $0.51_{-0.29}^{+0.19}$ & - & - \\
\hline & $\Delta \kappa_{\gamma}$ & $\begin{array}{r}-0.71_{-0.32}^{+0.39} \\
-0.39\end{array}$ & - & - \\
\hline & $\lambda_{\gamma}$ & $0.00_{-0.14}^{+0.17}$ & - & - \\
\hline \multirow{3}{*}{$q \bar{q} q \bar{q}$} & $\Delta g_{1}^{Z}$ & $-0.06_{-0.09}^{+0.10}$ & $-0.03_{-0.10}^{+0.11}$ & - \\
\hline & $\Delta \kappa_{\gamma}$ & $-0.11_{-0.27}^{+0.30}$ & $0.21_{-0.78}^{+0.34}$ & - \\
\hline & $\lambda_{\gamma}$ & $-0.15_{-0.10}^{+0.11}$ & $-0.02+0.14$ & - \\
\hline \multirow{3}{*}{$\ell \nu \ell \nu$} & $\Delta g_{1}^{Z}$ & $-0.17_{-0.20}^{+0.30}$ & - & - \\
\hline & $\Delta \kappa_{\gamma}$ & $-0.35_{-0.41}^{+0.80}$ & - & - \\
\hline & $\lambda_{\gamma}$ & $0.05_{-0.13}^{+0.13}$ & - & - \\
\hline
\end{tabular}

The $\mathrm{OO}_{1}$ method is used to measure the couplings $\Delta \mathrm{g}_{1}^{\mathrm{Z}}, \Delta \kappa_{\gamma}$ and $\lambda_{\gamma}$ for all five WW final states considered in this analysis, namely e $e q \bar{q}, \mu \nu q \bar{q}, \tau \nu q \bar{q}, q \bar{q} q \bar{q}$ and $\ell \nu \ell \nu$. The $\mathrm{OO}_{2}$ method is employed to measure the couplings $\Delta \mathrm{g}_{1}^{\mathrm{Z}}, \Delta \kappa_{\gamma}$ and $\lambda_{\gamma}$ for the $e \nu q \bar{q}, \mu \nu q \bar{q}$ and $q \bar{q} q \bar{q}$ channel.

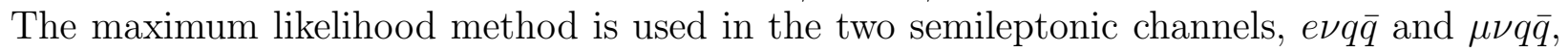
to measure the standard set of couplings $\Delta \mathrm{g}_{1}^{\mathrm{Z}}, \Delta \kappa_{\gamma}$ and $\lambda_{\gamma}$ and the real and imaginary parts of the C- or P-violating couplings $\mathrm{g}_{4}^{\mathrm{V}}, \mathrm{g}_{5}^{\mathrm{V}}, \tilde{\kappa}_{\mathrm{V}}$, and $\tilde{\lambda}_{\mathrm{V}}$, where $\mathrm{V}$ denotes either $\gamma$ or $\mathrm{Z}$.

A comparison of the three couplings $\Delta \mathrm{g}_{1}^{\mathrm{Z}}, \Delta \kappa_{\gamma}$ and $\lambda_{\gamma}$, using the three methods $\mathrm{OO}_{1}$, $\mathrm{OO}_{2}$ and maximum likelihood, is given in Table 5. For $\mathrm{OO}_{1}$ and $\mathrm{OO}_{2}$, the combined results at 183 and $189 \mathrm{GeV}$ are extracted by adding up the corresponding $\chi^{2}$ terms, while for the maximum likelihood method results are extracted by summing up the corresponding $\log L$ functions. The error intervals for each coupling are defined as the $68 \%$ confidence intervals obtained by integration of the likelihood functions, to accommodate cases with non-parabolic behaviour of the log-likelihood function.

The linearity of the three fitting procedures is checked by repeating the fits using Monte 
Table 6: The expected error for the three methods, $\mathrm{OO}_{1}, \mathrm{OO}_{2}$ and maximum likelihood, at $189 \mathrm{GeV}$ for the three couplings $\Delta \mathrm{g}_{1}^{\mathrm{Z}}, \Delta \kappa_{\gamma}$ and $\lambda_{\gamma}$. The maximum likelihood method has only been applied in the two semileptonic channels, $e \nu q \bar{q}$ and $\mu \nu q \bar{q}$.

\begin{tabular}{|c|c||c|c|c|}
\hline \multirow{2}{*}{ Channel } & \multicolumn{1}{|c||}{ Coupling } & \multicolumn{3}{c|}{ Expected error } \\
\cline { 4 - 5 }$e \nu q \bar{q}$ & $\Delta \mathrm{OO}_{1}$ & $\mathrm{OO}_{2}$ & LL \\
\hline \multirow{5}{*}{$\mu \kappa_{\gamma}$} & 0.11 & 0.12 & 0.11 \\
& $\lambda_{\gamma}$ & 0.48 & 0.43 & 0.40 \\
& $\lambda_{\gamma}$ & 0.13 & 0.12 & 0.13 \\
\hline \multirow{3}{*}{$\mu \nu q \bar{q}$} & $\Delta \mathrm{g}_{1}^{\mathrm{Z}}$ & 0.11 & 0.11 & 0.10 \\
& $\Delta \kappa_{\gamma}$ & 0.48 & 0.41 & 0.37 \\
& $\lambda_{\gamma}$ & 0.11 & 0.11 & 0.13 \\
\hline
\end{tabular}

Carlo event samples generated with non-zero values for the TGCs. In all cases the results are consistent to those values within the statistical uncertainty of the Monte Carlo samples.

The consistency of the Monte Carlo simulations with the data is verified by comparisons of the distributions of the input quantities to the selections for data and Monte Carlo. In addition, the stability of the analysis with respect to the event selection is tested by varying the main selection criteria within reasonable limits. In no case significant discrepancies are found.

The reliability of the errors from each fitting procedure is investigated by performing fits to a large number of independent Monte Carlo samples, each corresponding to the integrated luminosity of the data. These samples, typically 300 , are then reweighted to non-zero values for the TGCs and passed through the analysis chain. The expected $68 \%$ confidence levels, obtained from the distributions of the fit values, show good correspondence with the $68 \%$ confidence intervals obtained for data.

The consistency of the data results from the three different methods used for the $e \nu q \bar{q}$ and $\mu \nu q \bar{q}$ channels, has been checked by performing fits to a large number of independent Monte Carlo samples. The results obtained with the three different methods are compatible and the expected spread between the methods show a good agreement with the observed differences in the data.

The expected $68 \%$ confidence level intervals, obtained from the distributions of the fit values for the three methods at $189 \mathrm{GeV}$ for the three couplings $\Delta \mathrm{g}_{1}^{\mathrm{Z}}, \Delta \kappa_{\gamma}$ and $\lambda_{\gamma}$, are listed in Table 6. The expected errors of the $\mathrm{OO}_{2}$ and the maximum likelihood method are very similar, whereas the $\mathrm{OO}_{1}$ method is slightly worse in the case of $\Delta \kappa_{\gamma}$. For the final results, the $\mathrm{OO}_{2}$ method is therefore employed in the analysis of the $e \nu q \bar{q}, \mu \nu q \bar{q}$ and $q \bar{q} q \bar{q}$ final states and the $\mathrm{OO}_{1}$ analysis is used for the remaining $\tau \nu q \bar{q}$ and $\ell \nu \ell \nu$ final states.

\subsubsection{Systematic uncertainties}

In the following the different sources of systematic errors for each decay channel and their determination for the various methods are briefly described. The different contributions of each source to the total systematic error for the three couplings $\Delta \mathrm{g}_{1}^{\mathrm{Z}}, \Delta \kappa_{\gamma}$ and $\lambda_{\gamma}$, as 
obtained with the OO methods, are given for each channel in Table 7. The systematic uncertainties for the combined $e \nu q \bar{q}$ and $\mu \nu q \bar{q}$ channels are listed in Table 8 for the C- or P-violating couplings, determined with the maximum likelihood method.

The following sources, listed in the approximate relative importance, have been considered to be fully correlated between the channels:

- Fragmentation: The effect of fragmentation in hadronic W decays is estimated by fitting the couplings in samples of events generated with KORALW where the default JETSET fragmentation is replaced by HERWIG [36] fragmentation. The HERWIG fragmentation parameters are tuned at the $\mathrm{Z}$ using hadronic events with flavour tagging [29].

- $\mathbf{W}^{+} \mathbf{W}^{-}$cross section: The uncertainty due to the theoretical error on the expected $\mathrm{W}^{+} \mathrm{W}^{-}$cross section predicted by KORALW is estimated by changing the $\mathrm{W}^{+} \mathrm{W}^{-}$cross section by $\pm 2 \%[9]$.

- Luminosity: The effect of the error on the integrated luminosity is estimated by varying the measured integrated luminosity by $\pm 0.7 \%$ [37.

- LEP energy: The uncertainty on the LEP energy affects the determination of the couplings via the kinematic fitting procedure and the cross section measurement. The values of the LEP energy are varied in the range $\pm 0.050 \mathrm{GeV}$ [38], which has a negligible effect on the results.

- W mass: The analysis is repeated using Monte Carlo samples generated at different values of the $W$ mass to investigate the effects due to the uncertainty of $\pm 62 \mathrm{MeV}$ in the $\mathrm{W}$ mass measured at hadron colliders [39].

- Calorimeter absolute scale: The absolute energy scale of the electromagnetic and hadronic calorimeters is determined using hadronic $\mathrm{Z}$ events. The uncertainties in the absolute scale are found to be $\pm 0.9 \%$ and $\pm 2 \%$ for the electromagnetic and hadronic calorimeter, respectively. The effect of a possible miscalibration of the calorimeters is evaluated on Monte Carlo samples by scaling the electromagnetic and hadronic part of the measured energy independently by these amounts. The largest of the observed shifts for each calorimeter is combined in quadrature.

- Particle tracking: The definition of a good charged track has been tightened in this analysis in order to minimise possible effects from residual tracking distortions primarily in the forward regions of the detector. Corrections for the distortions are determined by studying $\mathrm{Z} \rightarrow \mu^{+} \mu^{-}$events, and possible remaining distortions have been estimated using Bhabha events. The systematic uncertainty related to tracking is assessed by applying the corrections and adding the remaining distortions independently to Monte Carlo event samples and repeating the analysis 28].

- Jet energy corrections: Detailed comparisons of reconstructed jets in Monte Carlo and data are used to parametrise small corrections to Monte Carlo jet energies as function of the jet polar angle to the beam axis [28]. In order to evaluate the effect from the uncertainty in the Monte Carlo jet energy corrections, two alternative correction functions, corresponding to $\pm 1 \sigma$ errors of the discrepancies, are used. The largest shift with respect to the nominal correction is taken as the systematic error [28]. 
- Higher order terms: The effect from missing higher order terms, $\mathcal{O}\left(\alpha^{3}\right)$, in the simulation of initial state radiation in the KORALW generator, is assessed following the procedure described in Ref. [28]. In summary, the error on the couplings is determined by comparing fits of Monte Carlo samples with events weighted to $\mathcal{O}(\alpha) / \mathcal{O}\left(\alpha^{2}\right)$ with fits to the corresponding unweighted samples, which have been generated in the second order leading-log approximation. Recently, new improved calculations with the Double Pole Approximation [40] have appeared. The improved CC03 cross section and angular distributions predicted by two independent Monte Carlo programs, RacoonWW 41] and YFSWW [42], could introduce small changes on the couplings. For the time being these effects have not been included.

Errors assumed to be uncorrelated between channels include:

- Bose-Einstein correlations: The effect of Bose-Einstein correlations in the $q \bar{q} q \bar{q}$ channel is investigated by repeating the analysis on Monte Carlo events generated with KORALW and fragmented using JETSET with Bose-Einstein correlations for all particles, following the implementation in LUBOEI [43]. The scheme for restoring four-momentum conservation denoted $\mathrm{BE}_{3}$, which has been tuned to the LEP1 Z data, is considered [29].

- Colour reconnection: The uncertainty arising from possible colour reconnection effects is assessed by studying Monte Carlo implementations of different colour reconnection scenarios in the parton evolution scheme in JETSET [44. The analysis is repeated with $q \bar{q} q \bar{q}$ events generated with the EXCALIBUR generator and hadronised with and without colour reconnection in the model referred to as SK1, as described in [28]. The systematic error is taken as the difference in fitted couplings from samples without colour reconnection and with colour reconnection in about $30 \%$ of the events.

- Background estimation: The error on the couplings from the uncertainties in the background estimation is evaluated by varying the normalisation of the main background processes. The background from QCD is changed by $\pm 5 \%$ based on comparisons between data and Monte Carlo simulation. The background from $\gamma \gamma$, Zee and ZZ processes is varied by $\pm 30 \% \pm 20 \%$ and $\pm 2 \%$, respectively, to account for the theoretical uncertainty in the prediction for those processes [9].

- Monte Carlo statistics: The effect of the Monte Carlo statistics is included in the systematic uncertainty.

- Jet charge assignment: To investigate the effects from the uncertainties on the jet charge, the reconstructed $\mathrm{W}$ charge is shifted by 0.01 . This number is based on comparisons between data and Monte Carlo simulation ( $\mathrm{Z}$ peak data) [45]. The effect on the couplings is found to be negligible.

The systematic uncertainties listed above as fully correlated between channels are also assumed to be fully correlated between years. In addition, the systematic errors from BoseEinstein correlations and colour reconnection are taken to be to fully correlated between years.

For both optimal observable methods, $\mathrm{OO}_{1}$ and $\mathrm{OO}_{2}$, the systematic errors have been calculated based on the changes in the mean values of the respective observables. This is incorporated in the TGC extraction by including the corresponding covariance matrix for 


\begin{tabular}{|c|c|c|c|c|c|c|c|c|c|c|c|c|c|c|c|}
\hline & \multicolumn{5}{|c|}{$\Delta g_{1}^{Z}$} & \multicolumn{5}{|c|}{$\Delta \kappa_{\gamma}$} & \multicolumn{5}{|c|}{$\lambda_{\gamma}$} \\
\hline Source & $e \nu q \bar{q}$ & $\mu \nu q \bar{q}$ & $\tau \nu q \bar{q}$ & $q \bar{q} q \bar{q}$ & $\ell \nu \ell \nu$ & $e \nu q \bar{q}$ & $\mu \nu q \bar{q}$ & $\tau \nu q \bar{q}$ & $q \bar{q} q \bar{q}$ & $\ell \nu \ell \nu$ & $e \nu q \bar{q}$ & $\mu \nu q \bar{q}$ & $\tau \nu q \bar{q}$ & $q \bar{q} q \bar{q}$ & $\ell \nu \ell \nu$ \\
\hline \multicolumn{16}{|l|}{ Correlated errors } \\
\hline Fragmentation & 0.01 & 0.01 & 0.09 & 0.02 & - & 0.06 & 0.04 & 0.20 & 0.05 & - & 0.01 & - & 0.15 & 0.04 & - \\
\hline $\mathrm{W}^{+} \mathrm{W}^{-}$cross section & - & 0.02 & 0.10 & 0.02 & 0.03 & - & 0.15 & 0.09 & 0.04 & 0.07 & - & 0.02 & - & 0.03 & - \\
\hline Luminosity & - & - & 0.05 & - & 0.01 & - & 0.03 & 0.04 & 0.03 & 0.03 & - & - & - & 0.01 & 0.01 \\
\hline LEP energy & - & - & - & - & - & - & - & - & 0.01 & - & - & - & - & - & - \\
\hline $\mathrm{W}$ mass & - & - & 0.03 & - & 0.02 & 0.02 & - & 0.17 & 0.02 & 0.10 & - & - & 0.06 & - & 0.03 \\
\hline Calorimeter scale & - & - & 0.12 & - & 0.03 & 0.03 & - & 0.12 & - & 0.04 & 0.01 & - & 0.03 & - & 0.03 \\
\hline Tracking & - & - & - & & 0.04 & - & - & - & - & 0.06 & - & - & - & - & 0.01 \\
\hline Jet corrections & - & - & 0.01 & - & - & - & - & 0.01 & 0.02 & - & - & - & - & - & - \\
\hline Higher order terms & - & - & 0.01 & - & - & - & - & 0.01 & - & 0.01 & - & - & 0.14 & - & - \\
\hline \multicolumn{16}{|l|}{ Uncorrelated errors } \\
\hline Bose-Einstein correlations & - & - & - & 0.01 & - & - & - & - & 0.02 & - & - & - & - & 0.01 & - \\
\hline Colour reconnection & - & - & - & - & - & - & - & - & 0.02 & - & - & - & - & 0.01 & - \\
\hline Background estimation & - & - & - & 0.01 & - & - & - & - & 0.02 & - & - & - & - & 0.01 & - \\
\hline Monte Carlo statistics & - & - & 0.07 & & 0.24 & - & - & 0.19 & & - & - & - & 0.17 & - & 0.06 \\
\hline Total & 0.01 & 0.02 & 0.20 & 0.03 & 0.25 & 0.07 & 0.16 & 0.36 & 0.08 & 0.15 & 0.01 & 0.02 & 0.27 & 0.05 & 0.07 \\
\hline
\end{tabular}

Table 7: Summary of systematic uncertainties for the couplings $\Delta \mathrm{g}_{1}^{\mathrm{Z}}, \Delta \kappa_{\gamma}$ and $\lambda_{\gamma}$. A description of the different sources is given in the text. Systematic uncertainties below 0.005 are indicated by a dash. 


\begin{tabular}{|c|c|c|c|c|c|c|c|c|c|c|c|c|c|c|c|c|}
\hline & \multicolumn{8}{|c|}{ Real } & \multicolumn{8}{|c|}{ Imaginary } \\
\hline Source & $\tilde{\kappa}_{\gamma}$ & $\bar{\lambda}_{\gamma}$ & $\tilde{\kappa}_{Z}$ & $\tilde{\lambda}_{Z}$ & $g_{4}^{\gamma}$ & $g_{5}^{\gamma}$ & $g_{4}^{Z}$ & $g_{5}^{Z}$ & $\tilde{\kappa}_{\gamma}$ & $\tilde{\lambda}_{\gamma}$ & $\tilde{\kappa}_{Z}$ & $\tilde{\lambda}_{Z}$ & $g_{4}^{\gamma}$ & $g_{5}^{\gamma}$ & $g_{4}^{Z}$ & $g_{5}^{Z}$ \\
\hline \multicolumn{17}{|l|}{ Correlated errors } \\
\hline Fragmentation & 0.04 & 0.03 & 0.01 & - & 0.04 & 0.01 & 0.03 & 0.05 & 0.02 & 0.02 & 0.01 & 0.01 & 0.01 & 0.07 & - & 0.02 \\
\hline $\mathrm{W}^{+} \mathrm{W}^{-}$cross section & 0.02 & 0.02 & 0.01 & 0.01 & - & 0.02 & - & - & 0.01 & 0.01 & 0.01 & - & 0.04 & 0.02 & 0.02 & 0.01 \\
\hline Luminosity & 0.01 & 0.01 & - & - & 0.01 & 0.02 & - & - & - & - & - & - & 0.01 & 0.02 & 0.01 & 0.01 \\
\hline LEP energy & 0.01 & - & - & - & 0.02 & 0.04 & 0.01 & 0.03 & 0.01 & 0.01 & - & - & 0.01 & 0.02 & - & 0.02 \\
\hline $\mathrm{W}$ mass & - & - & - & - & - & 0.02 & - & - & - & - & - & - & 0.01 & 0.01 & 0.01 & - \\
\hline Calorimeter scale & 0.01 & 0.01 & 0.01 & 0.01 & - & 0.04 & - & 0.03 & 0.01 & 0.01 & - & - & 0.01 & 0.01 & - & 0.01 \\
\hline Tracking & 0.02 & 0.01 & 0.01 & 0.01 & - & 0.02 & - & - & 0.01 & 0.01 & - & - & 0.01 & 0.02 & 0.01 & - \\
\hline Jet corrections & - & - & - & - & 0.01 & 0.01 & 0.01 & 0.01 & - & - & - & - & - & 0.01 & - & 0.01 \\
\hline Higher order terms & - & - & - & - & - & - & - & - & - & - & - & - & - & - & - & - \\
\hline \multicolumn{17}{|l|}{ Uncorrelated errors } \\
\hline Monte Carlo statistic & 0.02 & 0.02 & 0.02 & 0.01 & 0.04 & 0.04 & 0.03 & 0.02 & 0.01 & 0.01 & 0.01 & 0.01 & 0.03 & 0.05 & 0.02 & 0.03 \\
\hline Background estimation & - & - & - & - & - & - & - & - & - & - & - & - & - & - & - & - \\
\hline Total & 0.06 & 0.05 & 0.02 & 0.02 & 0.06 & 0.08 & 0.04 & 0.07 & 0.03 & 0.02 & 0.02 & 0.01 & 0.05 & 0.09 & 0.04 & 0.04 \\
\hline
\end{tabular}

Table 8: Summary of systematic uncertainties for the combined $e \nu q \bar{q}$ and $\mu \nu q \bar{q}$ channels for C- or P-violating couplings. A description of the different sources is given in the text. Systematic uncertainties below 0.005 are indicated by a dash. 
Table 9: The combined results for 183 and $189 \mathrm{GeV}$ for each $\mathrm{W}^{+} \mathrm{W}^{-}$decay channel for the three couplings $\Delta \mathrm{g}_{1}^{\mathrm{Z}}, \Delta \kappa_{\gamma}$ and $\lambda_{\gamma}$. The error includes the statistical and systematic uncertainty.

\begin{tabular}{|c||r|r|r|}
\hline \multicolumn{1}{|c||}{} & \multicolumn{1}{c|}{ Coupling } \\
\hline Channel & \multicolumn{1}{c|}{$\Delta \mathrm{g}_{1}^{Z}$} & \multicolumn{1}{c|}{$\lambda_{\gamma}$} \\
\hline$e \nu q \bar{q}$ & $0.09_{-0.09}^{+0.09}$ & $0.46_{-0.32}^{+0.33}$ & $0.21_{-0.10}^{+0.11}$ \\
$\mu \nu q \bar{q}$ & $0.01_{-0.10}^{+0.10}$ & $0.20_{-0.34}^{+0.64}$ & $-0.08_{-0.09}^{+0.09}$ \\
$\tau \nu q \bar{q}$ & $0.51_{-0.37}^{+0.21}$ & $-0.71_{-0.39}^{+0.54}$ & $0.00_{-0.15}^{+0.18}$ \\
$q \bar{q} q \bar{q}$ & $-0.03_{-0.10}^{+0.10}$ & $0.27_{-0.26}^{+0.30}$ & $0.01_{-0.12}^{+0.13}$ \\
$\ell \nu \ell \nu$ & $-0.17_{-0.21}^{+0.36}$ & $-0.35_{-0.41}^{+0.82}$ & $0.05_{-0.13}^{+0.14}$ \\
\hline
\end{tabular}

the systematic uncertainties. By this procedure the systematic uncertainties are folded with the proper statistical correlations between the optimal observables and the results of the fits include both the statistical and systematic errors. The systematic uncertainties listed in Table 7 are derived from the changes in the optimal observables mean values. They are not used as such in the analysis but serve only as a representation of the systematic contributions from the different sources.

The systematic uncertainties for the maximum likelihood method are convoluted into the $\log L$ functions by assuming parabolic behaviour of the systematic errors around the fitted TGC value.

\subsubsection{Final results from $\mathrm{W}$-pair production}

The combined results from all $\mathrm{W}^{+} \mathrm{W}^{-}$decay channels at 183 and $189 \mathrm{GeV}$ for the three couplings $\Delta \mathrm{g}_{1}^{\mathrm{Z}}, \Delta \kappa_{\gamma}$ and $\lambda_{\gamma}$, are obtained by combining the $\mathrm{OO}_{2}$ analysis of the $e \nu q \bar{q}, \mu \nu q \bar{q}$ and $q \bar{q} q \bar{q}$ final states with the $\mathrm{OO}_{1}$ analysis of the $\tau \nu q \bar{q}$ and $\ell \nu \ell \nu$ final states. The correlation of the systematic errors between the different channels and energies are included as described in Section. 6.3.2. The results for $\Delta \mathrm{g}_{1}^{\mathrm{Z}}, \Delta \kappa_{\gamma}$ and $\lambda_{\gamma}$, including systematic uncertainties, are listed in Table 9. The final $68 \%$ and $95 \%$ combined W-pair result for the three couplings $\Delta \mathrm{g}_{1}^{\mathrm{Z}}, \Delta \kappa_{\gamma}$ and $\lambda_{\gamma}$ is summarised in Table 10. The corresponding $\log L$ curves, including systematic uncertainties, are shown in Figure 8.

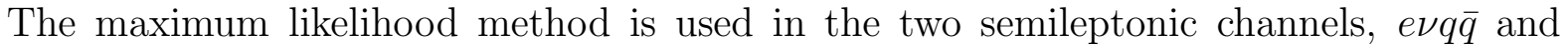
$\mu \nu q \bar{q}$, to measure the real and imaginary parts of the C- or P-violating couplings $\mathrm{g}_{4}^{\mathrm{V}}, \mathrm{g}_{5}^{\mathrm{V}}, \tilde{\kappa}_{\mathrm{V}}$, and $\tilde{\lambda}_{\mathrm{V}}$, where $\mathrm{V}$ denotes either $\gamma$ or $\mathrm{Z}$. The combined 183 and $189 \mathrm{GeV}$ results, including the systematic uncertainties, for the C- or P-violating couplings are summarised in Table 10.

In all cases described above, each coupling is determined fixing the other couplings to their Standard Model values. The error intervals for each coupling are defined as the $68 \%$ confidence level intervals obtained by integration of the likelihood functions, to accommodate cases with non-parabolic behaviour of the log-likelihood function. 
Table 10: Combined 183 and $189 \mathrm{GeV}$ W-pair results for the three C- and P-conserving couplings, $\Delta \mathrm{g}_{1}^{\mathrm{Z}}, \Delta \kappa_{\gamma}$ and $\lambda_{\gamma}$, and the $\mathrm{C}$ - or P-violating couplings. The error includes the statistical and systematic uncertainty. The corresponding $95 \%$ confidence level intervals are listed in the last column.

\begin{tabular}{|c||r|c|}
\hline & fit result & $95 \%$ confidence limits \\
\hline$\Delta \mathrm{g}_{1}^{Z}$ & $0.02_{-0.06}^{+0.06}$ & {$[-0.09,0.14]$} \\
\hline$\Delta \kappa_{\gamma}$ & $0.22_{-0.20}^{+0.21}$ & {$[-0.15,0.66]$} \\
\hline$\lambda_{\gamma}$ & $0.04_{-0.06}^{+0.06}$ & {$[-0.08,0.17]$} \\
\hline \hline $\operatorname{Re}\left(\tilde{\kappa}_{\gamma}\right)$ & $-0.19_{-0.17}^{+0.19}$ & {$[-0.51,0.18]$} \\
\hline $\operatorname{Re}\left(\tilde{\lambda}_{\gamma}\right)$ & $0.17_{-0.16}^{+0.14}$ & {$[-0.15,0.43]$} \\
\hline $\operatorname{Re}\left(\tilde{\kappa}_{Z}\right)$ & $-0.09_{-0.11}^{+0.12}$ & {$[-0.30,0.14]$} \\
\hline $\operatorname{Re}\left(\tilde{\lambda}_{Z}\right)$ & $0.07_{-0.10}^{+0.09}$ & {$[-0.12,0.25]$} \\
\hline $\operatorname{Re}\left(g_{4}^{\gamma}\right)$ & $0.06_{-0.35}^{+0.34}$ & {$[-0.62,0.72]$} \\
\hline $\operatorname{Re}\left(g_{5}^{\gamma}\right)$ & $-0.02_{-0.51}^{+0.51}$ & {$[-1.02,0.98]$} \\
\hline $\operatorname{Re}\left(g_{4}^{Z}\right)$ & $0.07_{-0.23}^{+0.23}$ & {$[-0.38,0.50]$} \\
\hline $\operatorname{Re}\left(g_{5}^{Z}\right)$ & $-0.06_{-0.31}^{+0.32}$ & {$[-0.67,0.56]$} \\
\hline $\operatorname{Im}\left(\tilde{\kappa}_{\gamma}\right)$ & $0.10_{-0.12}^{+0.12}$ & {$[-0.14,0.33]$} \\
\hline $\operatorname{Im}\left(\tilde{\lambda}_{\gamma}\right)$ & $-0.08_{-0.10}^{+0.10}$ & {$[-0.27,0.11]$} \\
\hline $\operatorname{Im}\left(\tilde{\kappa}_{Z}\right)$ & $0.03_{-0.08}^{+0.08}$ & {$[-0.13,0.19]$} \\
\hline $\operatorname{Im}\left(\tilde{\lambda}_{Z}\right)$ & $-0.03_{-0.07}^{+0.07}$ & {$[-0.16,0.10]$} \\
\hline $\operatorname{Im}\left(g_{4}^{\gamma}\right)$ & $0.37_{-0.30}^{+0.30}$ & {$[-0.23,0.95]$} \\
\hline $\operatorname{Im}\left(g_{5}^{\gamma}\right)$ & $-0.01_{-0.56}^{+0.57}$ & {$[-1.10,1.10]$} \\
\hline $\operatorname{Im}\left(g_{4}^{Z}\right)$ & $0.27_{-0.20}^{+0.20}$ & {$[-0.13,0.65]$} \\
\hline $\operatorname{Im}\left(g_{5}^{Z}\right)$ & $0.07_{-0.35}^{+0.35}$ & {$[-0.62,0.76]$} \\
\hline
\end{tabular}


Table 11: Combined results for $\Delta \mathrm{g}_{1}^{\mathrm{Z}}, \Delta \kappa_{\gamma}$ and $\lambda_{\gamma}$ from $\mathrm{W}^{+} \mathrm{W}^{-}$production at $172-189 \mathrm{GeV}$, single- $\gamma$ and single-W production at $161-189 \mathrm{GeV}$. The errors include systematic uncertainties. The corresponding $95 \%$ confidence level intervals are listed in the last column.

\begin{tabular}{|l||c|c|}
\hline Coupling & fit result & $95 \%$ confidence limits \\
\hline$\Delta \mathrm{g}_{1}^{\mathrm{Z}}$ & $0.023_{-0.055}^{+0.059}$ & {$[-0.087,0.141]$} \\
\hline$\Delta \kappa_{\gamma}$ & $0.022_{-0.115}^{+0.119}$ & {$[-0.200,0.258]$} \\
\hline$\lambda_{\gamma}$ & $0.040_{-0.052}^{+0.054}$ & {$[-0.062,0.147]$} \\
\hline
\end{tabular}

Table 12: Result of a three-parameter fit for $\Delta \mathrm{g}_{1}^{\mathrm{Z}}, \Delta \kappa_{\gamma}$ and $\lambda_{\gamma}$ using the combined information from $\mathrm{W}$-pair production at $172-189 \mathrm{GeV}$, single- $\gamma$ and single-W production at $161-189 \mathrm{GeV}$. The statistical and systematic uncertainties are combined in a $68 \%$ one-dimensional error. The corresponding correlations are given in the last column.

\begin{tabular}{|l||c||ccc|}
\hline \multicolumn{1}{|c||}{ Coupling } & \multicolumn{1}{c||}{ fit result } & \multicolumn{3}{c|}{ Correlation } \\
$\mathrm{g}_{1}^{\mathrm{Z}}$ & $0.013_{-0.068}^{+0.066}$ & 1.0 & -0.1 & -0.6 \\
\cline { 1 - 1 }$\Delta \kappa_{\gamma}$ & $0.043_{-0.110}^{+0.110}$ & & 1.0 & -0.1 \\
\cline { 1 - 1 }$\lambda_{\gamma}$ & $0.023_{-0.077}^{+0.074}$ & & & 1.0 \\
\hline
\end{tabular}

\section{Combined TGC results}

The measurements from single- $\gamma$, single $\mathrm{W}$ and $\mathrm{WW}$ production, are combined with previous ALEPH results from $\mathrm{W}^{+} \mathrm{W}^{-}$production at $172 \mathrm{GeV}$ [1], single- $\mathrm{W}$ production at $183 \mathrm{GeV}$ [3] and single- $\gamma$ production at 183 [4. The combined results are listed in Table 11. In Figure 9 the corresponding one-parameter $\log L$ curves are shown.

To study the full correlation between the parameters, two- and three-parameter fits, where two or all three couplings are allowed to vary, are also presented. The fits use the combined information from $\mathrm{W}$-pair production, single- $\mathrm{W}$ production and single- $\gamma$ production at $183-189 \mathrm{GeV}$.

For the three parameter fit the results and the errors computed from a variation from the minimum of the $\log L$ functions of 0.5 , are summarised in Table 12 including the systematic uncertainties. The correlation matrix of the three-parameter fit is also given in Table 12. This correlation matrix is evaluated at the local minimum, and the correlations vary substantially depending on the exact value of the minimum. The projections onto the two dimensional plane of the three dimensional envelope of the $95 \%$ confidence level volume, representing the integration of the confidence over the corresponding third coupling, are shown in Figure 10 . The $95 \%$ confidence limits of the respective 2-parameter fits of the three pairs of couplings $\left(\Delta \mathrm{g}_{1}^{\mathrm{Z}}, \Delta \kappa_{\gamma}\right),\left(\Delta \mathrm{g}_{1}^{\mathrm{Z}}, \lambda_{\gamma}\right)$ and $\left(\Delta \kappa_{\gamma}, \lambda_{\gamma}\right)$ are shown as full lines. The systematic uncertainties are included in the limits shown. No deviations from the Standard Model expectations are observed. 


\section{Summary and conclusions}

The triple gauge-boson couplings have been measured using W-pair events at 183 and $189 \mathrm{GeV}$, single-W production at $189 \mathrm{GeV}$ and single- $\gamma$ production at $189 \mathrm{GeV}$. Combining with previous ALEPH results from $\mathrm{W}^{+} \mathrm{W}^{-}$production at $172 \mathrm{GeV}$, single-W production and single- $\gamma$ production at $183 \mathrm{GeV}$, the three couplings $\Delta \mathrm{g}_{1}^{\mathrm{Z}}, \Delta \kappa_{\gamma}$ and $\lambda_{\gamma}$ have been measured individually, assuming the two other couplings to be fixed at their Standard Model value. The results are

$$
\begin{array}{llr}
\Delta \mathrm{g}_{1}^{\mathrm{Z}}= & 0.023_{-0.055}^{+0.059} \\
\Delta \kappa_{\gamma}= & 0.022_{-0.115}^{+0.119} \\
\lambda_{\gamma}= & 0.040_{-0.052}^{+0.054}
\end{array},
$$

where the error includes systematic uncertainties. The corresponding $95 \%$ confidence level limits,

$$
\begin{aligned}
& -0.087<\Delta \mathrm{g}_{1}^{\mathrm{Z}}<0.141 \\
& -0.200<\Delta \kappa_{\gamma}<0.258 \\
& -0.062<\lambda_{\gamma}<0.147,
\end{aligned}
$$

are in good agreement with the Standard Model expectation. Multi-parameter fits, where two or all three couplings are allowed to vary show also good agreement with the Standard Model.

In addition, semileptonic $\mathrm{W}$-pair events were used to set limits on the $\mathrm{C}$ - or P-violating couplings $\mathrm{g}_{4}^{\mathrm{V}}, \mathrm{g}_{5}^{\mathrm{V}}, \tilde{\kappa}_{\mathrm{V}}$, and $\tilde{\lambda}_{\mathrm{V}}$, where $\mathrm{V}$ denotes either $\gamma$ or Z. No deviations from the Standard Model expectations are observed.

\section{Acknowledgements}

It is a pleasure to congratulate our colleagues from the CERN accelerator divisions for the highly successful operation of LEP at high energies. We are indebted to the engineers and technicians in all our institutions for the contributions to the excellent performance of ALEPH. Those of us from non-member countries thank CERN for its hospitality. 


\section{A Leptonic Neural Network Input Variables}

The Neural Network (NN) calculates an approximation of the multidimensional probability density function in the following 13 input variables, for signal and backgrounds. The NN is applied, after preselection, to events with at least two opposite charged tracks with momentum - after bremsstrahlung correction - in excess of $15 \mathrm{GeV}$. The NN uses variables related to the lepton candidates, to the missing momentum, global event variables, and WW kinematics. They are listed here together with their relative discriminating power, namely the statistical correlation with the neural network output:

- $\operatorname{missing}$ mass squared $(13.7 \%)$;

- missing transverse momentum (11.5\%);

- angle between the two most energetic tracks (9.6\%);

- energy of the second most energetic track, $(8.7 \%)$;

- total energy found in a $12^{\circ}$ cone around the beam axis $(8.2 \%)$;

- number of identified leptons with an energy greater than $15 \mathrm{GeV}(8 \%)$;

- missing transverse momentum with respect to the plane defined by the beam axis and the 3D-thrust axis (7.8\%);

- energy of the most energetic track (7.4\%);

- invariant mass of the two most energetic tracks $(6.6 \%)$;

- missing longitudinal momentum (6.3\%);

- scalar sum of the transverse components of the two most energetic tracks with respect to a 2D-thrust axis, built from the projection of the track momenta on the transverse plane $(5.4 \%)$;

- number of isolated neutral clusters with energy more than $4 \mathrm{GeV}$ outside a cone of $10^{\circ}$ around each of the two most energetic tracks and forming an invariant mass with each of them of more than $2 \mathrm{GeV}(4.1 \%)$;

- cosine of the angle between the most energetic track and the axis perpendicular to the plane defined by the second most energetic track and the z-axis $(2.6 \%)$. 


\section{References}

[1] ALEPH Collaboration, Measurement of Triple Gauge-Boson Couplings at 172 GeV, Phys. Lett. B422 (1998) 369.

[2] DELPHI Collaboration, Measurement and interpretation of the $W$-pair cross-section in $e^{+} e^{-}$interactions at 161 GeV, Phys. Lett. B397 (1997) 158;

DELPHI Collaboration, Measurement of Trilinear Gauge Couplings in $e^{+} e^{-}$Collisions at $161 \mathrm{GeV}$ and $172 \mathrm{GeV}$, Phys. Lett. B423 (1998) 194;

DELPHI Collaboration, Measurements of the Trilinear Gauge Boson Couplings WWV ( $V \equiv \gamma, Z)$ in $e^{+} e^{-}$Collisions at 183 GeV, Phys. Lett. B459 (1999) 382;

L3 Collaboration, Pair-Production of $W$ Bosons in $e^{+} e^{-}$Interactions at $\sqrt{s}=161 \mathrm{GeV}$, Phys. Lett. B398 (1997) 223;

L3 Collaboration, Measurements of Mass, Width and Gauge Couplings of the W Boson at LEP, Phys. Lett. B413 (1997) 176;

L3 Collaboration, Measurement of Triple-Gauge-Boson Couplings of the $W$ Boson at LEP, Phys. Lett. B467 (1999) 171;

OPAL Collaboration, Measurement of the Triple Gauge Boson Coupling $\alpha_{W \phi}$ from $W^{+} W^{-}$Production in $e^{+} e^{-}$Collisions at $\sqrt{s}=161$ GeV, Phys. Lett. B397 (1997) 147;

OPAL Collaboration, Measurement of triple Gauge Boson Couplings from $W^{+} W^{-}$ Production at $\sqrt{s}=172$ GeV, Eur. Phys. J. C2 (1998) 597;

OPAL Collaboration, $W^{+} W^{-}$production and triple gauge boson couplings at LEP energies up to 183 GeV, Eur. Phys. J. C8 (1999) 191;

OPAL Collaboration, Measurement of $W$ Boson Polarisations and CP-violating Triple Gauge Couplings from $W^{+} W^{-}$Production at LEP, CERN-EP-2000-113 (submitted to Eur. Phys. J. C);

OPAL Collaboration, Measurement of triple gauge boson couplings from $W^{+} W^{-}$ production at LEP energies up to 189 GeV, CERN-EP-2000-114 (submitted to Eur. Phys. J. C).

[3] ALEPH Collaboration, A study of single $W$ Production in $e^{+} e^{-}$collisions at $\sqrt{s}=161$ 183 GeV, Phys. Lett. B462 (1999) 389.

[4] ALEPH Collaboration, Measurement of triple gauge WW couplings at LEP2 using photonic events, Phys. Lett. B445 (1998) 239.

[5] L3 Collaboration, Production of Single W Bosons at LEP, Phys. Lett. B403 (1997) 168; L3 Collaboration, Production of Single $W$ Bosons in $e^{+} e^{-}$Interactions at $130 \mathrm{GeV}$ $\leq \sqrt{s} \leq 183$ GeV and Limits on Anomalous $W W \gamma$ Couplings, Phys. Lett. B436 (1998) 417 ;

L3 Collaboration, Production of Single $W$ Bosons at $\sqrt{s}=189$ GeV and Measurement of WWr Gauge Couplings, Phys. Lett. B487 (2000) 229.

[6] CDF Collaboration, Observation of $W^{+} W^{-}$Production in $p \bar{p}$ Collisions at $\sqrt{s}=1.8$ TeV, Phys. Rev. Lett. 78 (1997) 4536;

DØ Collaboration, Studies of $W W$ and $W Z$ Production and Limits on Anomalous $W W \gamma$ and WWZ Couplings, Phys. Rev. D60 (1999) 072002.

[7] K. Hagiwara, R. D. Peccei, D. Zeppenfeld and K. Hikasa, Nucl. Phys. B282 (1987) 253. 
[8] M. Bilenky, J.L. Kneur, F.M. Renard and D. Schildknecht, Nucl. Phys. B409 (1993) 22.

[9] G. Gounaris, J.-L. Kneur and D. Zeppenfeld, from Physics at LEP2, CERN 96-01 p. 525, editors G. Altarelli, T. Sjöstrand and F. Zwirner.

[10] A. De Rújula, M. B. Gavela, P. Hernandez and E. Massó, Nucl. Phys. B384 (1992) 3.

[11] J. Ellison and J. Wudka, Ann. Rev. Nucl. Part. Sci. 48 (1998) 33.

[12] F. Boudjema et al., Phys. Rev. D43 (1991) 2223.

[13] ALEPH Collaboration, ALEPH: A Detector for Electron-Positron Annihilations at LEP, Nucl. Instrum. and Methods A 294 (1990) 121.

[14] ALEPH Collaboration, Performance of the ALEPH Detector at LEP, Nucl. Instrum. and Methods A 360 (1995) 481.

[15] S. Jadach, B.F.L. Ward and Z. Wạs, Comp. Phys. Commun. 79 (1994) 503.

[16] D. R. Yennie, S. C. Frautsch and H. Suura, Ann. Phys. (NY) 13 (1961) 379.

[17] G. Montagna et al., Nucl. Phys. B541 (1999) 31.

[18] J. Fujimoto et al., Comp. Phys. Commun. 100 (1997) 128.

[19] K. Hagiwara et al., Nucl. Phys. B365 (1991) 544.

[20] E. Barberio et al., Comp. Phys. Commun. 67 (1991) 115, Comp. Phys. Commun. 79 (1994) 291.

[21] S. Jadach et al., Comp. Phys. Commun. 70 (1992) 69, Comp. Phys. Commun. 76 (1993) 361.

[22] M. Skrzypek, S. Jadach, W. Placzek and Z. Wa̧s, Comp. Phys. Commun. 94 (1996) 216.

[23] T. Sjöstrand, Comp. Phys. Commun. 82 (1994) 74.

[24] ALEPH Collaboration, An Experimental Study of $\gamma \gamma \rightarrow$ Hadrons at LEP, Phys. Lett. B313 (1993) 509;

J. A. M. Vermasseren in Procedings of the IVth International Workshop on GammaGamma Interactions, Eds. G. Cochard and P. Kessler, Springer Verlag (1980).

[25] H. Anlauf et al., Comp. Phys. Commun. 79 (1994) 466.

[26] ALEPH Collaboration, Single- and multi-photon production in $e^{+} e^{-}$collisions at a centre-of-mass energy of 183 GeV, Phys. Lett. B429 (1998) 201.

[27] T. Tsukamoto and Y. Kurihara, Phys. Lett. B389 (1996) 162.

[28] ALEPH Collaboration, Measurement of the $W$ mass in $e^{+} e^{-}$collisions at 183 GeV, Phys. Lett. B453 (1999) 121.

[29] ALEPH Collaboration, Measurement of the W Mass and Width in $e^{+} e^{-}$collisions at 189 GeV, CERN-EP/2000-045 (submitted to Eur. Phys. J. C). 
[30] ALEPH Collaboration, Measurement of the $W$ mass in $e^{+} e^{-}$collisions at production threshold, Phys. Lett. B401 (1997) 347.

[31] ALEPH Collaboration, Measurement of the $W$-pair cross section in $e^{+} e^{-}$collisions at 172 GeV, Phys. Lett. B415 (1997) 435.

[32] ALEPH Collaboration, Measurement of W-pair cross section $e^{+} e^{-}$collisions at 183 GeV, Phys. Lett. B453 (1999) 107.

[33] M. Diehl and O. Nachtmann, Zeit. Phys. C62 (1994) 397.

[34] D.K. Fanourakis, D. Fassouliotis and S.E. Tzamarias, Nucl. Instrum. and Methods A412 (1998) 465; Nucl. Instrum. and Methods A414 (1998) 399.

[35] E. A. Kuraev and V. S. Fadin, Sov. J. Nucl. Phys. 41 (1985) 466.

[36] G. Marchesini et al., Comp. Phys. Commun. 67 (1992) 465.

[37] ALEPH Collaboration, Measurement of $W$-pair production in $e^{+} e^{-}$collisions at $189 \mathrm{GeV}$, CERN-EP/2000-052 (submitted to Phys. Lett. B).

[38] LEP energy working group, Evaluation of the LEP Centre-of-Mass energy above the WW production threshold, CERN-EP/98-191 (submitted to Eur. Phys. J. C);

LEP energy working group, Evaluation of the LEP Centre-of-Mass energy for data taken in 1998, LEP Energy Working Group Note 99/01.

[39] CDF Collaboration, A measurement of the $W$ boson mass, Phys. Rev. D52 (1995) 4784; DØ Collaboration, Measurement of the $W$ boson mass at the Fermilab p p collider, Phys. Rev. Lett. 80 (1998) 3008;

Y. K. Kim, in proceedings of the Lepton-Photon Symposium 1997, Hamburg, 28 July 1 August 1997.

[40] W. Beenakker, F. A. Berends and A. P. Chapowsky, Nucl. Phys. B548 (1999) 3.

[41] A. Denner, S. Dittmaier, M. Roth and D. Wackeroth, BI-TP 99/45, hep-ph/9912261 (1999).

[42] S. Jadach, W. Placzek, M. Skrzypek, B. F. L. Ward and Z. Wạs, Phys. Lett. B417 (1998) 326;CERN-TH/1999-222, UTHEP-98-0502.

[43] L. Lönnblad and T. Sjöstrand, Eur. Phys. J. C2 (1998) 165.

[44] T. Sjöstrand and V.A. Khoze, Zeit. Phys. C62 (1994) 281.

[45] ALEPH Collaboration, Determination of $\sin ^{2} \theta_{\text {eff }}$ Using Jet Charge Measurements in Hadronic Z Decays, Zeit. Phys. C71 (1996) 357. 
ALEPH
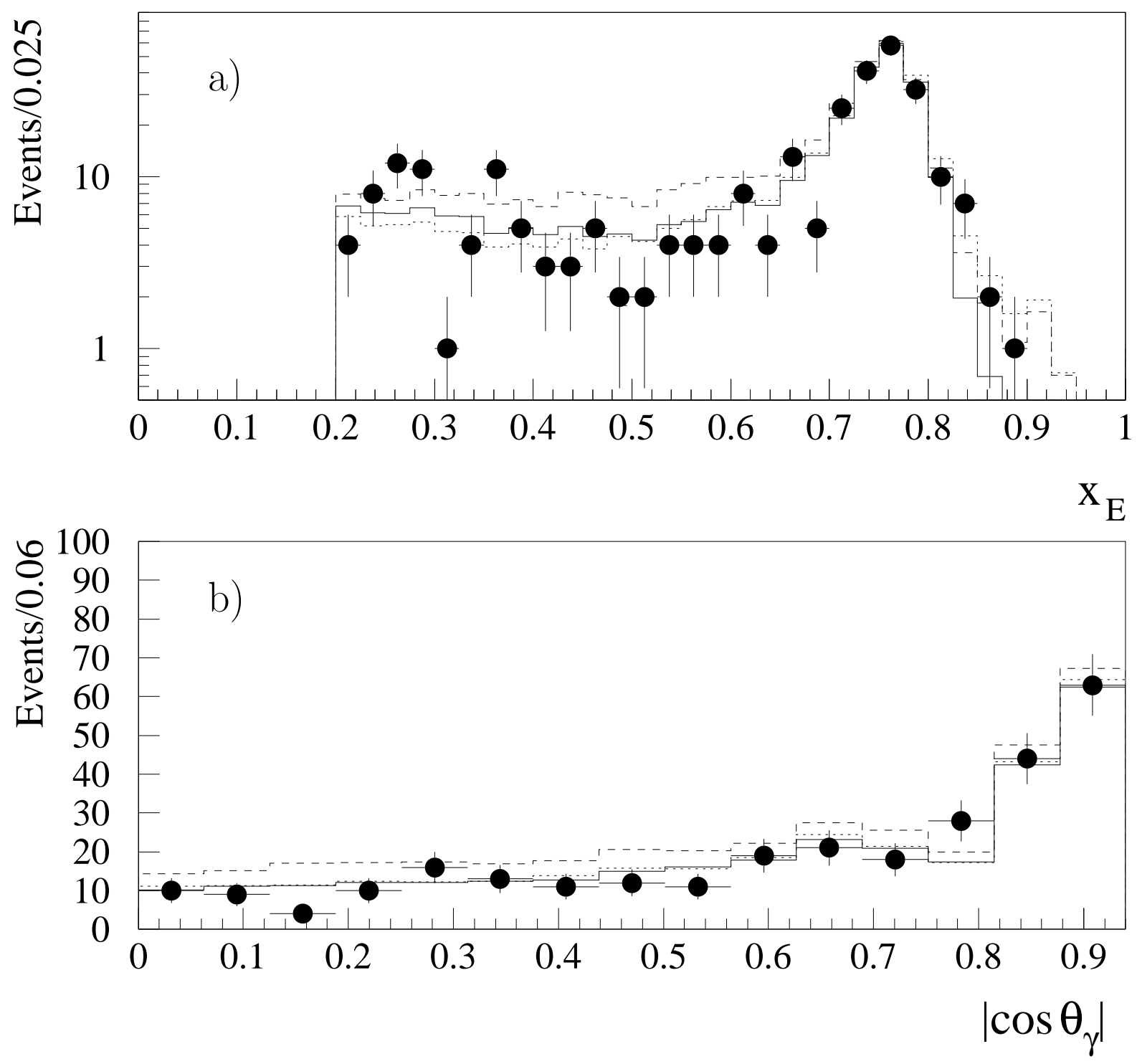

Figure 1: Distribution of a) the scaled energy, $x_{E}$, and b) the absolute value of the cosine of the polar angle for single- $\gamma$ events selected in $189 \mathrm{GeV}$ data. The data are represented by solid dots, while the solid histogram shows the distribution for the Standard Model. The dashed and dotted histograms show the distribution for non-standard values of $\Delta \kappa_{\gamma}= \pm 5.0$. 
ALEPH
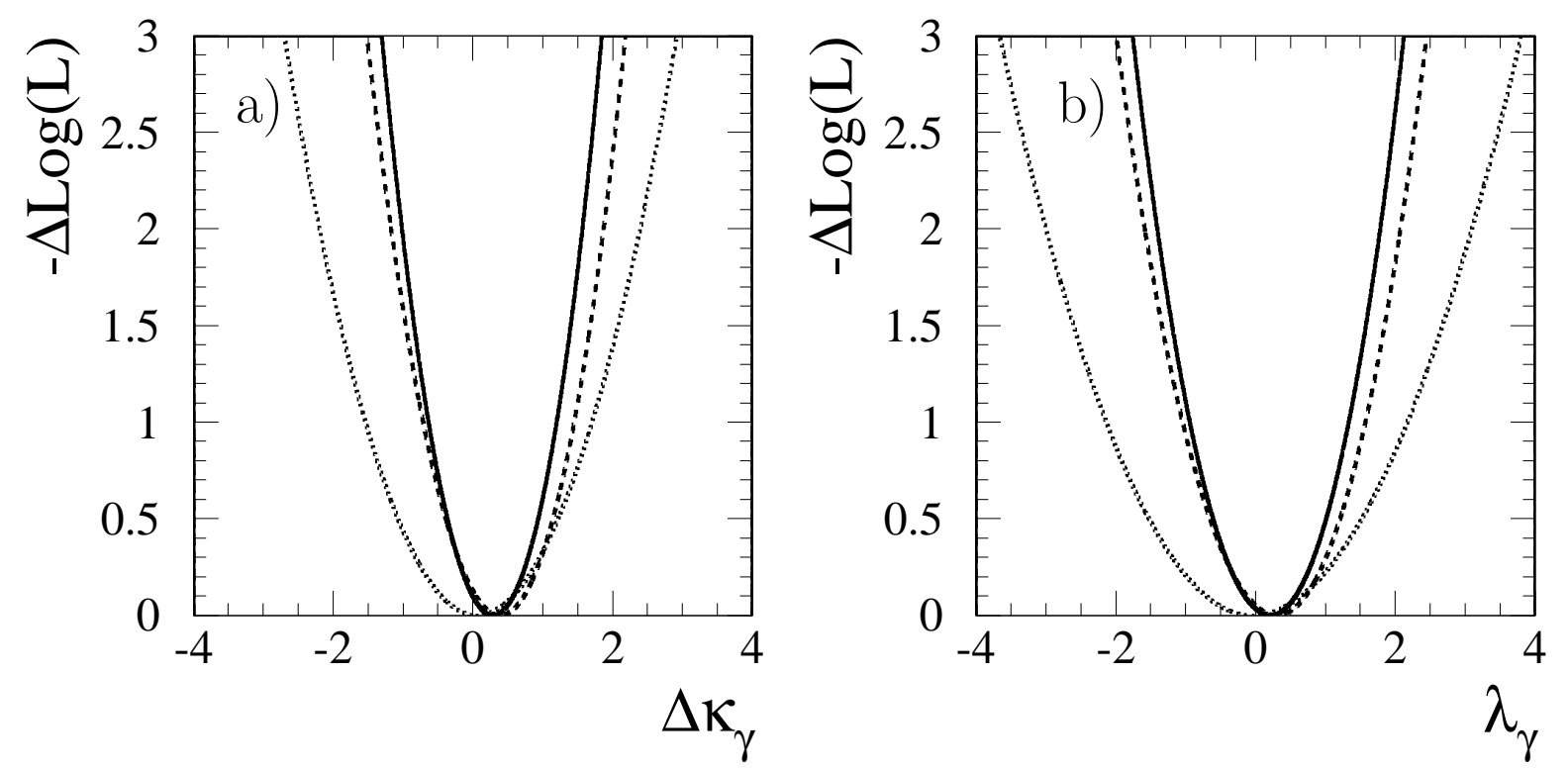

Figure 2: Negative log-likelihood curves, including systematic uncertainties, from the single$\gamma$ analysis for a) $\Delta \kappa_{\gamma}$ and b) $\lambda_{\gamma}$ for the $189 \mathrm{GeV}$ data (dashed line), 161 - $183 \mathrm{GeV}$ data [4] (dotted line), and the combined results (solid line). The curve for each coupling is obtained while fixing the other coupling to its Standard Model value. 


\section{ALEPH}
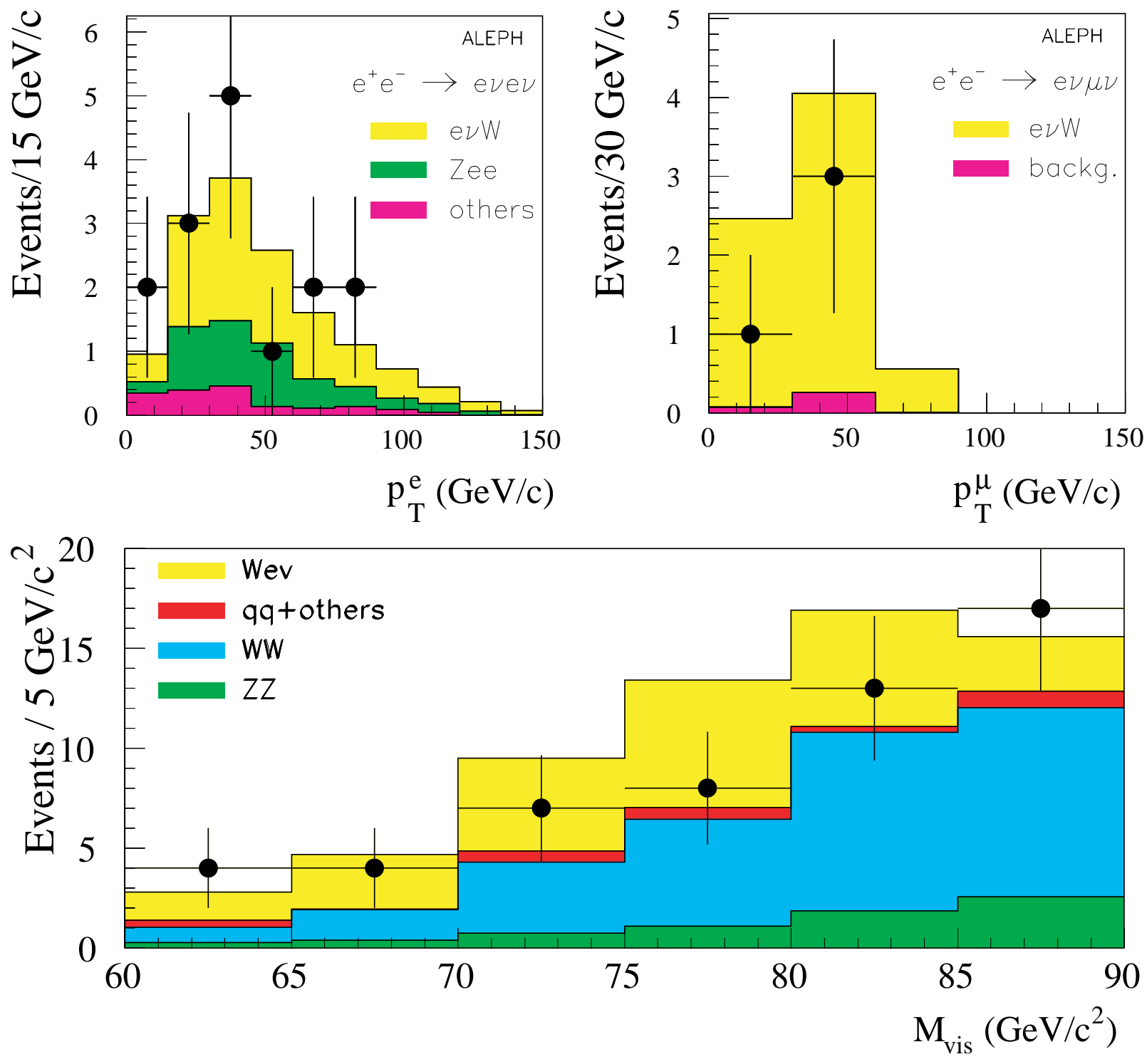

Figure 3: The distribution of the lepton transverse momentum, $p_{\mathrm{T}}^{l}$, for single-W events passing the final selection cuts for the leptonic electron (upper left) and muon (upper right) $\mathrm{W}$ decay. The lower plot shows the visible mass distribution from single- $\mathrm{W}$ events passing the final selection cuts for the hadronic $\mathrm{W}$ decay. The data are represented by the closed circles. The histograms correspond to the Standard Model prediction. 


\section{ALEPH}
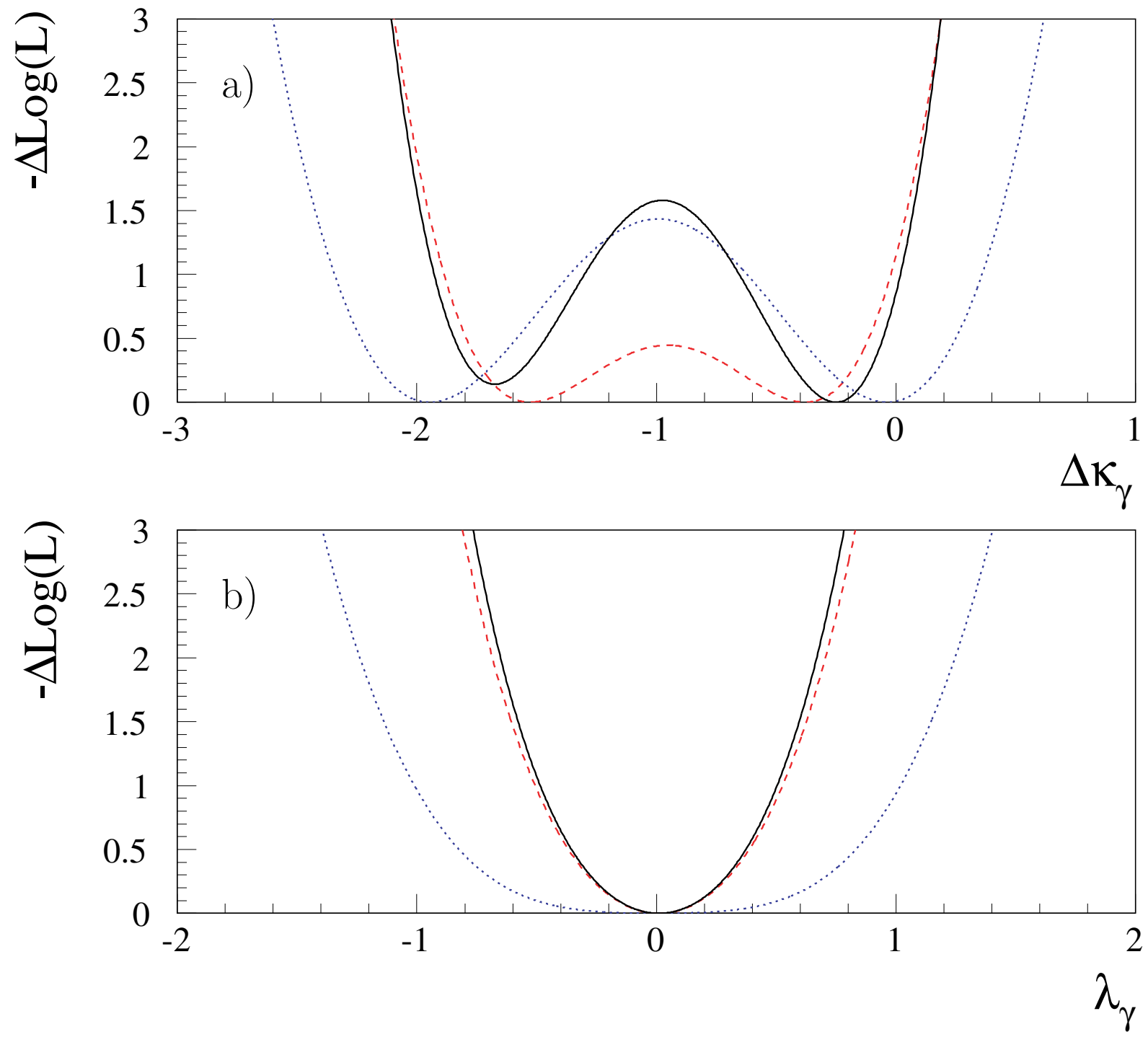

Figure 4: The negative log-likelihood curves from the single-W analysis as functions of a) $\Delta \kappa_{\gamma}$ and b) $\lambda_{\gamma}$ for the $189 \mathrm{GeV}$ data (dashed line), 161 - $183 \mathrm{GeV}$ data [刨] (dotted line) and the combined results (solid line). The curve for each coupling is obtained while fixing the other coupling to its Standard Model value. Systematic errors are not included in these curves. 


\section{ALEPH}
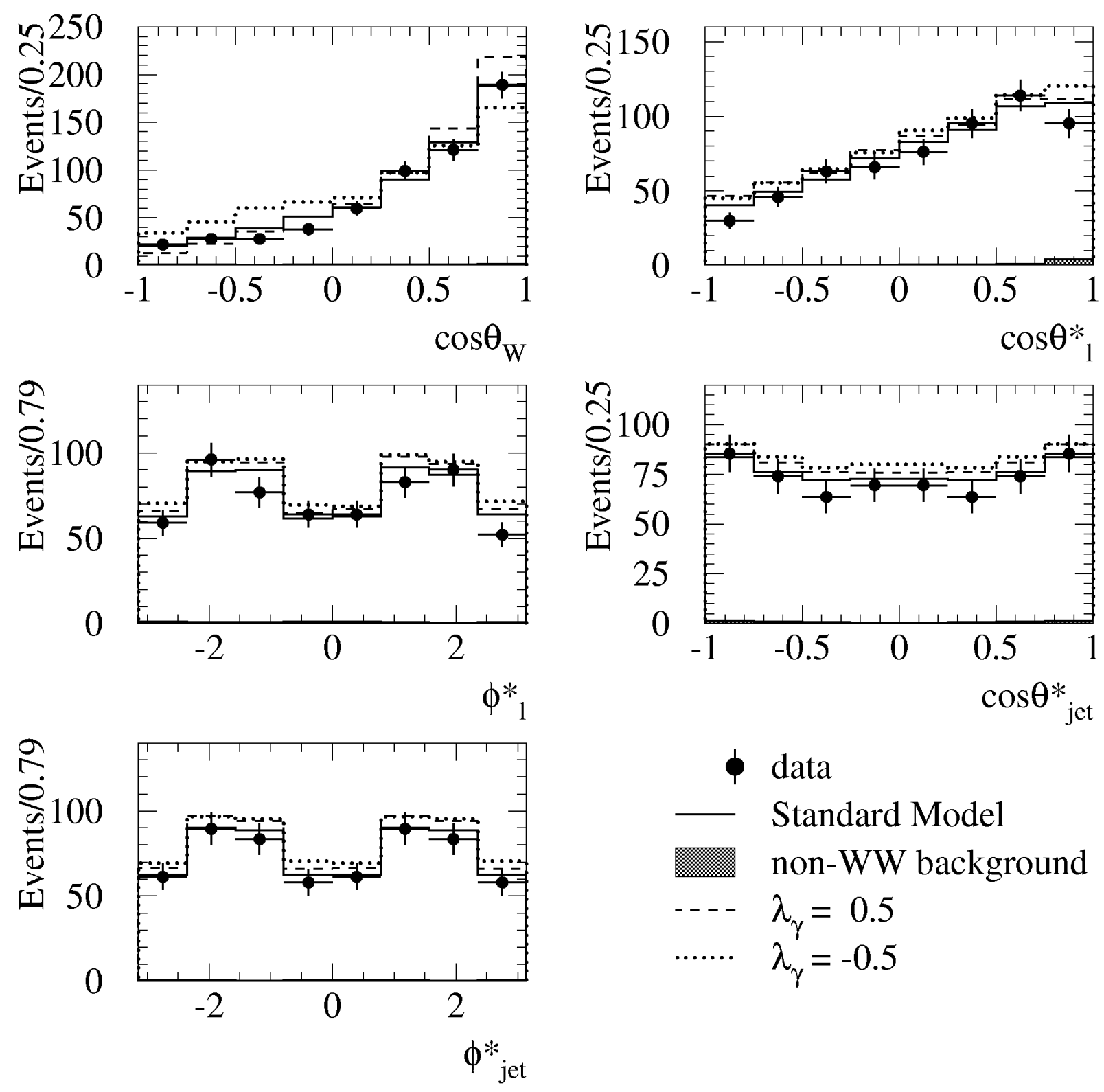

Figure 5: The distributions of the kinematic quantities $\cos \theta_{W}, \cos \theta_{l}^{*}, \phi_{l}^{*}, \cos \theta_{\text {jet }}^{*}$ and $\phi_{\text {jet }}^{*}$ from the combined sample of the $e \nu q \bar{q}$ and $\mu \nu q \bar{q}$ channels at $189 \mathrm{GeV}$. The measured variables are the angle $\theta_{W}$ between the $\mathrm{W}^{-}$and initial $e^{-}$in the $\mathrm{W}^{+} \mathrm{W}^{-}$rest frame, the polar and azimuthal angles of the lepton, $\theta_{1}^{*}$ and $\phi_{1}^{*}$, in the rest frame of its parent $\mathrm{W}$, and the polar and azimuthal angles of a quark jet, $\theta_{\text {jet }}^{*}$ and $\phi^{*}$ jet, in the rest frame of its parent W. As no quark flavour tagging is performed each of the two ambiguous solutions enters with a weight of 0.5. The data are represented by solid dots, while the solid and dashed histograms show distributions for Standard Model and non-standard values of $\lambda_{\gamma}= \pm 0.5$. 


\section{ALEPH}
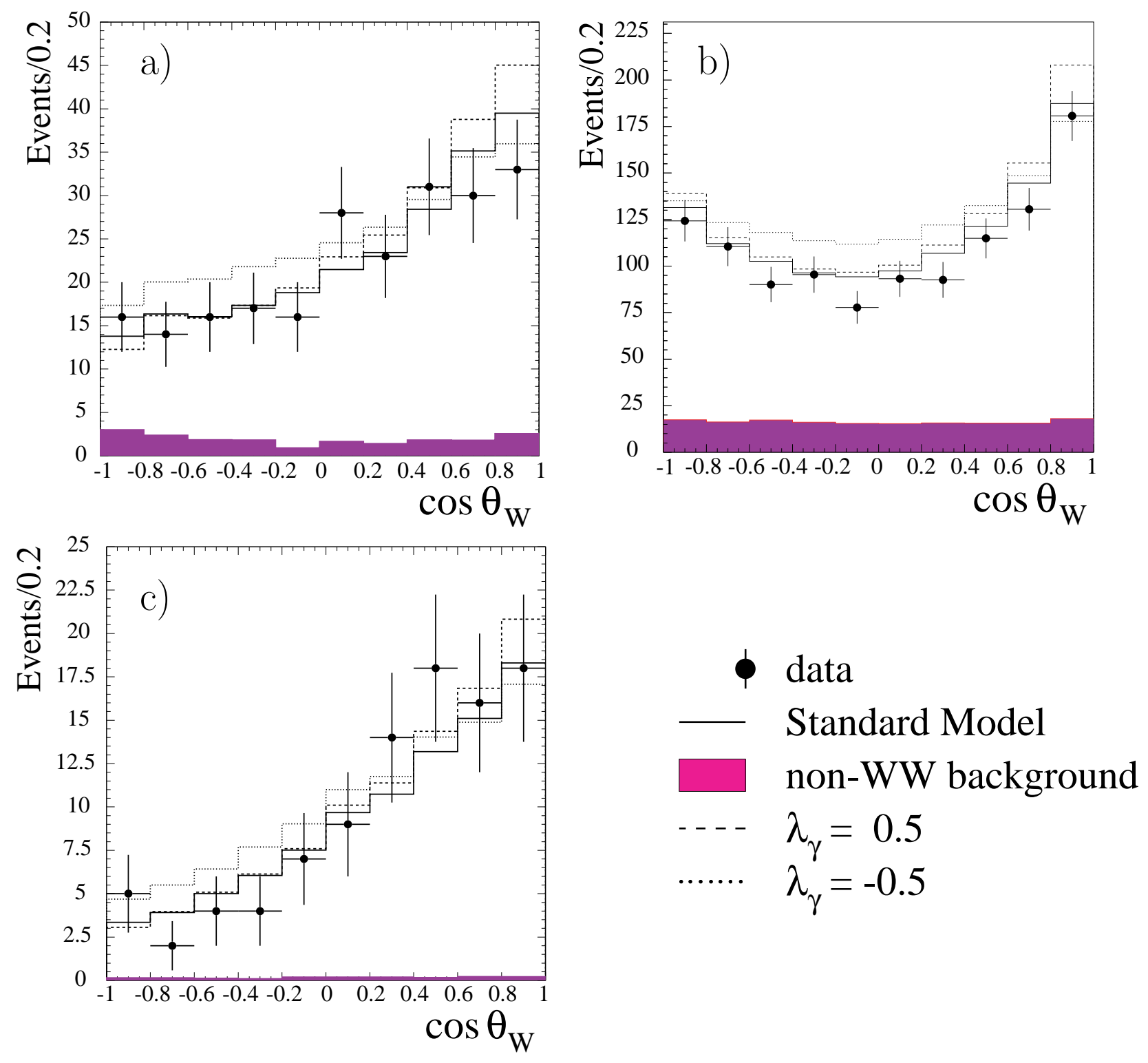

Figure 6: Distributions of the cosine of the $\mathrm{W}^{-}$production angle, $\cos \theta_{W}$, at $189 \mathrm{GeV}$ for a) $\tau \nu q \bar{q}$, b) $q \bar{q} q \bar{q}$ and c) $\ell \nu \ell \nu$ events. The data are represented by solid dots, while the solid and dashed histograms show distributions for Standard Model and non-standard values of the TGCs. The shaded area represents the non-WW background. For $q \bar{q} q \bar{q}$ events, each event enters with two solutions for $\cos \theta_{W}$ in the distribution with the weights $P_{+}$and $1-P_{+}$, where $P_{+}$is the probability for a di-jet pair to be a $\mathrm{W}^{+}$. For $\ell \nu \ell \nu$ events, each event enters with two solutions for $\cos \theta_{W}$ in the distribution with a weight of 0.5 . 


\section{ALEPH}
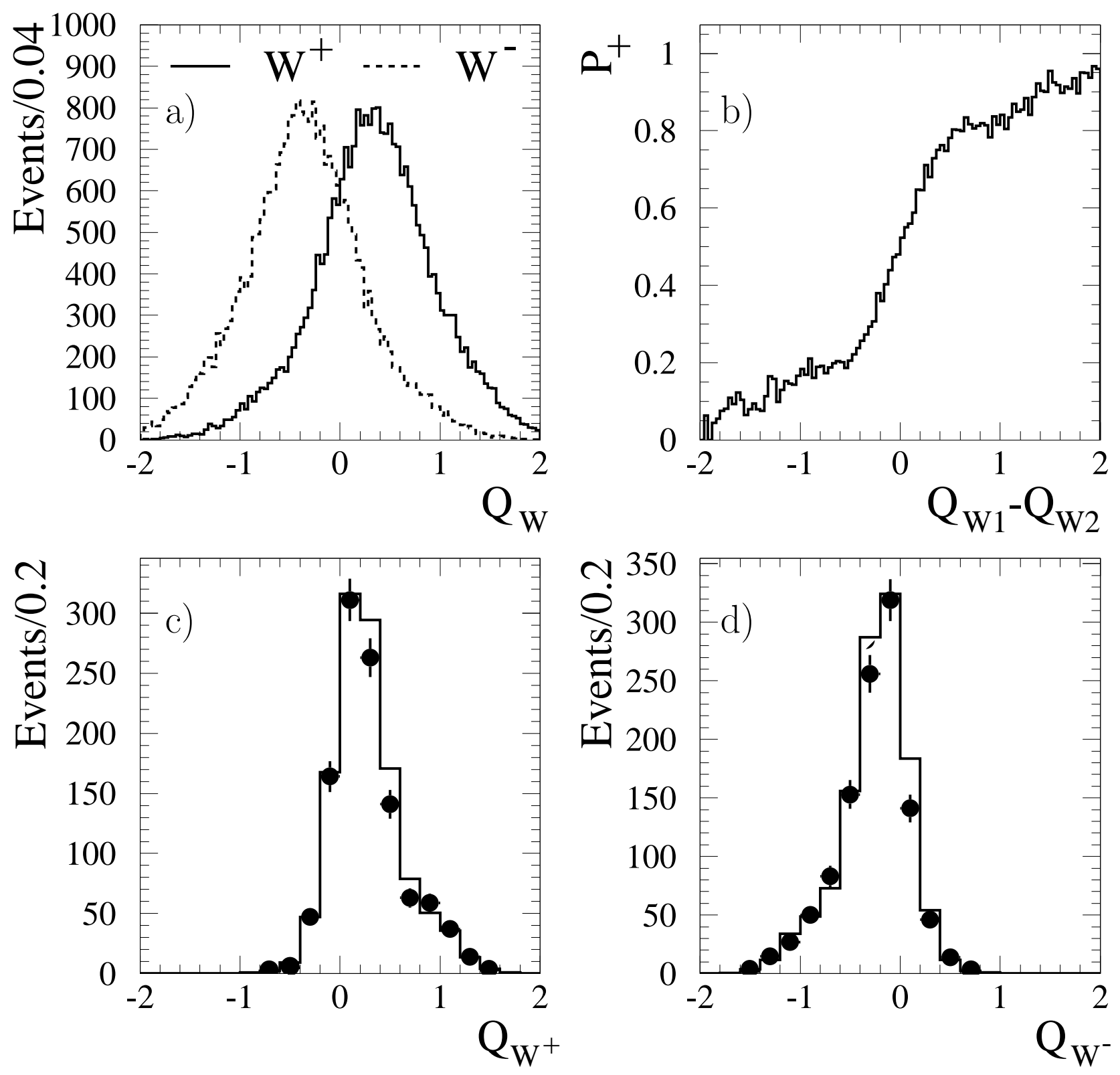

Figure 7: W-charge tagging distributions from $q \bar{q} q \bar{q} \mathrm{~W}$-pair events at $189 \mathrm{GeV}$. a) The distribution of the jet pair charge for $\mathrm{W}^{+}$(solid histogram) and $\mathrm{W}^{-}$(dashed histogram) decays for Monte Carlo events. b) The probability $P_{+}$as function of the charge difference between the two W's. c,d) Experimental distributions of $Q_{\mathrm{W}^{+}}$and $Q_{\mathrm{W}^{-}}$from semileptonic events. The data are represented by the dots and the Monte Carlo simulation by the histograms. The number of Monte Carlo events is normalised to the integrated luminosity of data. 


\section{ALEPH}
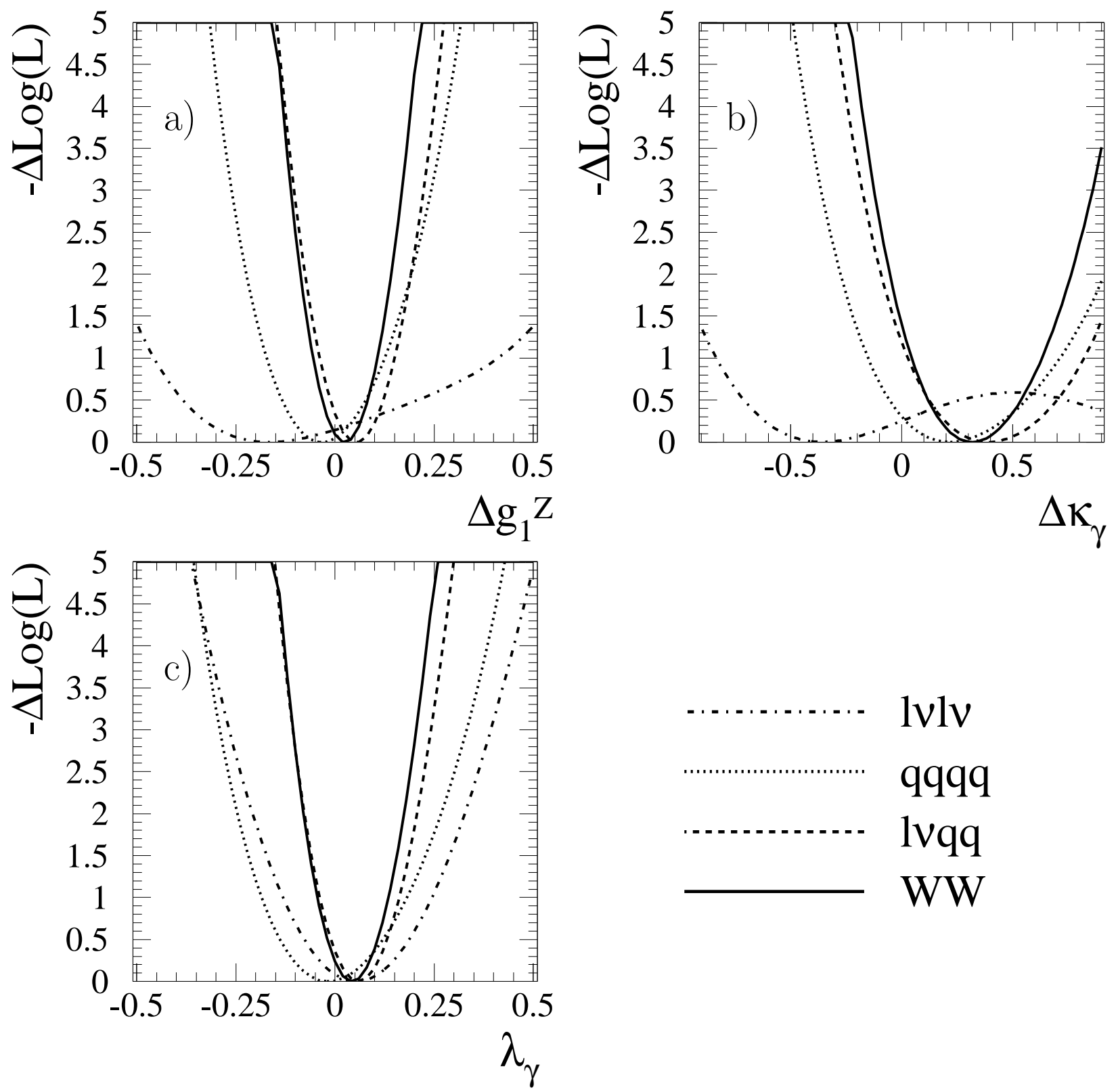

Figure 8: The combined negative log-likelihood curves from the $\mathrm{W}$-pair analysis of 183 and $189 \mathrm{GeV}$ data for the individual fits in the $\ell \nu q \bar{q}$ (dashed), $q \bar{q} q \bar{q}$ (dotted) and $\ell \nu \ell \nu$ (dasheddotted) channels for the three couplings a) $\Delta \mathrm{g}_{1}^{\mathrm{Z}}$, b) $\Delta \kappa_{\gamma}$ and c) $\lambda_{\gamma}$. The curve for each coupling is obtained while fixing the other couplings to their Standard Model value. The systematic uncertainties are included. The combined result for all channels is shown as the solid curve. 


\section{ALEPH}
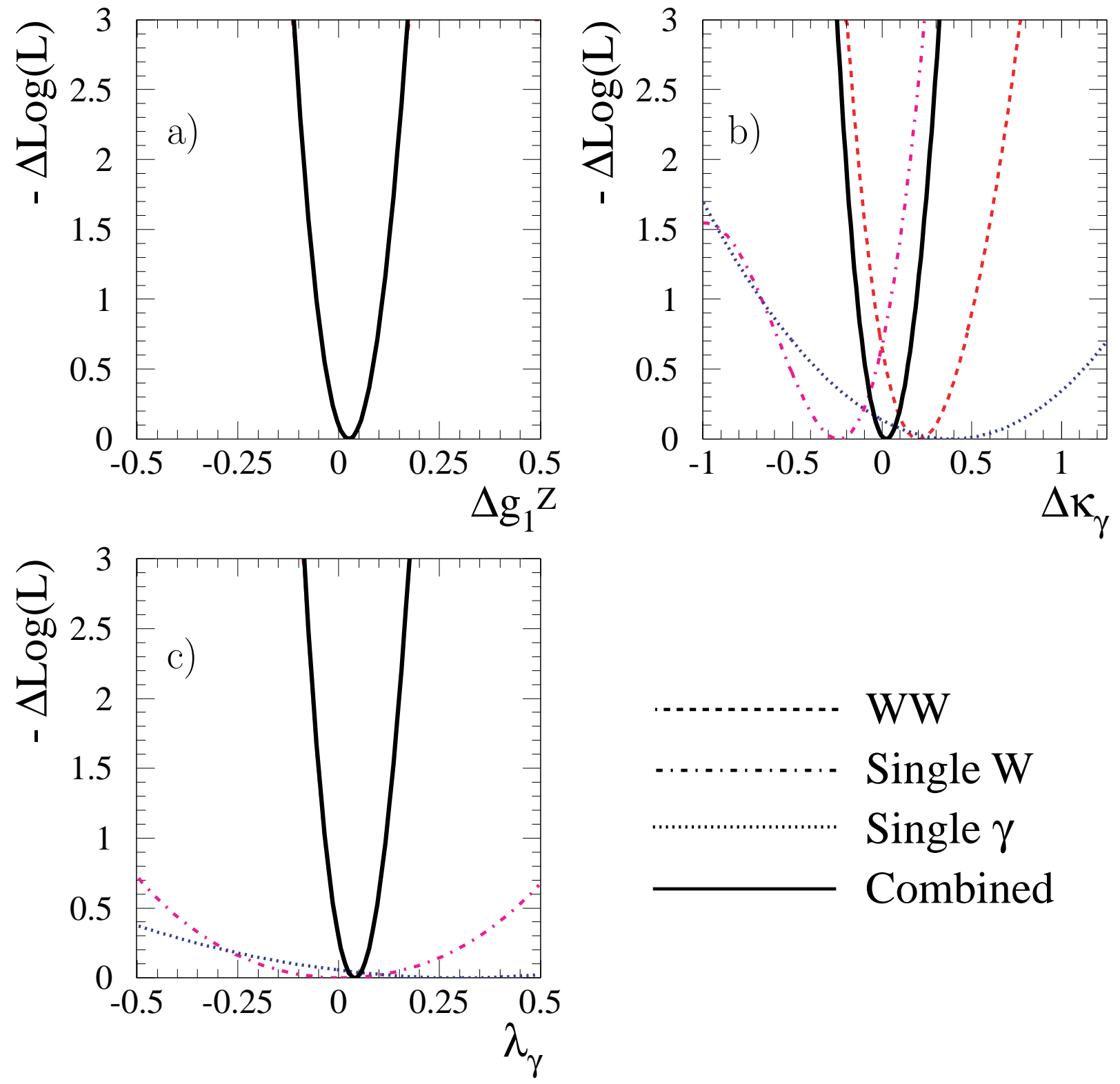

Figure 9: The negative log-likelihood curves for the combined fits using single- $\gamma$ (dotted), single-W (dashed-dotted) and $\mathrm{W}$-pair (dashed) production at energies up to $189 \mathrm{GeV}$ for the three couplings a) $\Delta \mathrm{g}_{1}^{\mathrm{Z}}$, b) $\Delta \kappa_{\gamma}$ and c) $\lambda_{\gamma}$. The curve for each coupling is obtained while fixing the other couplings to their Standard Model value. The systematic uncertainties are included. The combined result is shown as the solid curve. 


\section{ALEPH}
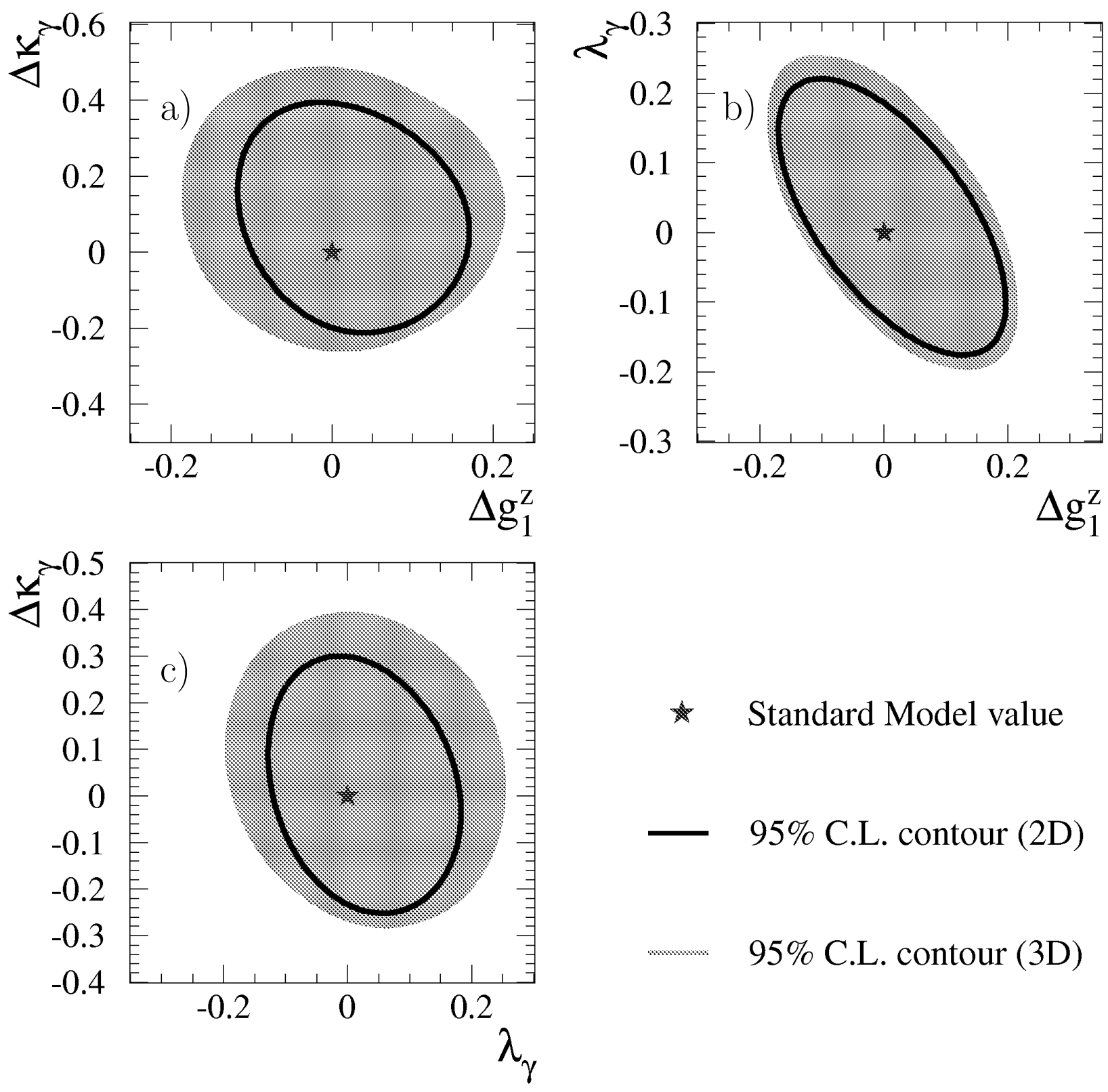

* Standard Model value

95\% C.L. contour (2D)

95\% C.L. contour (3D)

Figure 10: Multi-parameter fits using the combined data from single- $\gamma$, single-W and $\mathrm{W}$-pair production at energies up to $189 \mathrm{GeV}$. The two-dimensional $95 \%$ confidence level contours for the three pairs of couplings, a) $\left(\Delta \mathrm{g}_{1}^{Z}, \Delta \kappa_{\gamma}\right)$, b) $\left(\Delta \mathrm{g}_{1}^{Z}, \lambda_{\gamma}\right)$ and c) $\left(\Delta \kappa_{\gamma}, \lambda_{\gamma}\right)$. The solid lines show the $95 \%$ confidence level contours of the two-parameter fit. The shaded area is a projection onto the two-dimensional plane of the three-dimensional envelope of the $95 \%$ confidence level volume. The Standard Model point is represented by a star. 